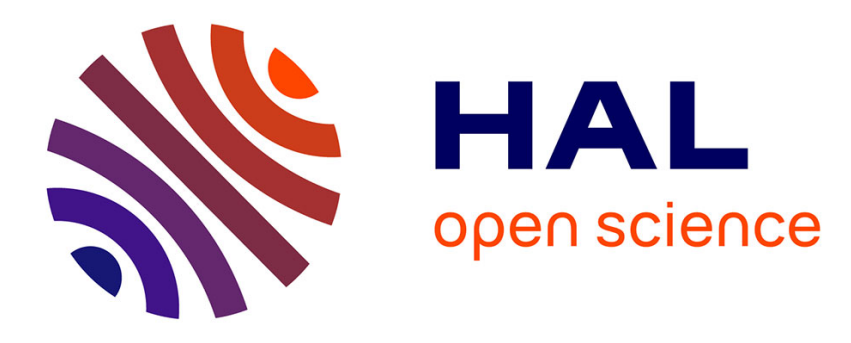

\title{
Weak Solutions for the Stationary Anisotropic and Nonlocal Compressible Navier-Stokes System
}

\author{
D. Bresch, Cosmin Burtea
}

\section{To cite this version:}

D. Bresch, Cosmin Burtea. Weak Solutions for the Stationary Anisotropic and Nonlocal Compressible Navier-Stokes System. 2020. hal-02502419v2

HAL Id: hal-02502419

https://hal.science/hal-02502419v2

Preprint submitted on 7 Apr 2020

HAL is a multi-disciplinary open access archive for the deposit and dissemination of scientific research documents, whether they are published or not. The documents may come from teaching and research institutions in France or abroad, or from public or private research centers.
L'archive ouverte pluridisciplinaire HAL, est destinée au dépôt et à la diffusion de documents scientifiques de niveau recherche, publiés ou non, émanant des établissements d'enseignement et de recherche français ou étrangers, des laboratoires publics ou privés. 


\title{
Weak Solutions for the Stationary Anisotropic and Nonlocal Compressible Navier-Stokes System
}

\author{
D. Bresch, $\quad$ C. Burtea ${ }^{\dagger}$
}

April 7, 2020

\begin{abstract}
In this paper, we prove existence of weak solutions for the stationary compressible Navier-Stokes equations with an anisotropic and nonlocal viscous stress tensor in a periodic domain $\mathbb{T}^{3}$. This gives an answer to an open problem important for applications in geophysics or in microfluidics. One of the key ingredients is the new identity discovered by the authors in [2] which was used to study the non-stationary anisotropic compressible Brinkman system.
\end{abstract}

\section{Introduction}

The stationary Navier-Stokes system for a barotropic compressible viscous fluid reads

$$
\left\{\begin{array}{l}
\operatorname{div}(\rho u)=0, \\
\operatorname{div}(\rho u \otimes u)-\mu \Delta u-(\mu+\lambda) \nabla \operatorname{div} u+\nabla p(\rho)=\rho f+g,
\end{array}\right.
$$

where $\mu$ and $\lambda$ are given positive constants representing the shear respectively the bulk viscosities, $f, g \in \mathbb{R}^{3}$ are given exterior forces acting on the fluid, $\rho \geq 0$ is the density, $p(\rho)=a \rho^{\gamma}$ represents the pressure, where $a>0$ and $\gamma \geq 1$ are given constants while $u \in \mathbb{R}^{3}$ is the velocity field. The total mass of the fluid is given i.e. the above system should be considered along with the equation

$$
\int_{\Omega} \rho=M>0
$$

where $M$ is given.

It is important to point out that all the known mathematical results regarding the existence of weak solutions for the stationary Navier-Stokes system strongly use the isotropic and local structure of the viscous stress tensor owing to the nice algebraic properties it induces for the so-called effective flux. Extending these results such as to take in account anisotropic or nonlocal viscous stress-tensors remained an open problem until now.

As explained in [16], one cannot expect that (1.1)-(1.2) with periodic boundary conditions to have a solution for any $f, g \in L^{\infty}$ because of the compatibility condition

$$
\int_{\mathbb{T}^{3}}(\rho f+g)=0
$$

which comes from integrating the momentum equation. Thus, if $f$ and $g$ have positive components this would imply that $\rho=0$ which clearly violates the total mass condition. One way to bypass this structural defect of the periodic case is to proceed as in [5] and consider forces $f$ that posses a certain symmetry which ensures the validity of (1.3). Another way to bypass this problem was suggested by P.L. Lions in [16] and consists in introducing the term $B \times(B \times u)$ with $B \in L^{\infty}\left(\mathbb{T}^{3}\right)$ a non-constant function in the momentum equation which can be interpreted as the effect of a magnetic field on the

\footnotetext{
${ }^{*}$ Univ. Grenoble Alpes, Univ. Savoie Mont-Blanc, CNRS, LAMA, Chambéry, France; didier.bresch@univ-smb.fr

${ }^{\dagger}$ Université Paris Diderot UFR Mathématiques Batiment Sophie Germain, Bureau 727, 8 place Aurélie Nemours, 75013 Paris; cburtea@math.univ-paris-diderot.fr
} 
fluid. We claim that the ideas presented in the present paper can be adapted to handle both situations but in order to avoid extra technical difficulties we choose to treat the case where $f=0$. We propose here to investigate the problem of existence of weak solutions $(\rho, u)$ for the following system:

$$
\left\{\begin{array}{l}
\operatorname{div}(\rho u)=0, \\
\operatorname{div}(\rho u \otimes u)-\mathcal{A} u+a \nabla \rho^{\gamma}=g
\end{array}\right.
$$

with

$$
\rho \geq 0, \quad \int_{\mathbb{T}^{3}} \rho(x) d x=M>0, \quad \int_{\mathbb{T}^{3}} u(x) d x=0,
$$

where the viscous diffusion operator $\mathcal{A}$ is given by

$$
\mathcal{A} \cdot=\underbrace{\mu \Delta \cdot+(\mu+\lambda) \nabla \operatorname{div}}_{\text {classical part }} \cdot+\underbrace{\mu \theta \partial_{33}}_{\text {anisotropic part }}+\underbrace{\eta * \Delta \cdot+\xi * \nabla \operatorname{div} \cdot}_{\text {nonlocal part }} .
$$

We will assume the following hypothesis $(\mathrm{H})$ :

- A given total mass $M>0$ of the fluid.

- An adiabatic constant $\gamma>3$ and a positive constant $a>0$.

- A forcing term $g$ such that

$$
g \in\left(L^{\frac{3(\gamma-1)}{2 \gamma-1}}\left(\mathbb{T}^{3}\right)\right)^{3} \quad \text { with } \int_{\mathbb{T}^{3}} g=0
$$

- The constant $\mu, \lambda$ and $\theta$ such that

$$
\mu, \mu+\lambda>0 \quad \text { and } \quad \theta>-1 .
$$

- The functions $\eta$ and $\xi$ satisfying

$$
\min \{1,1+\theta\} \mu-\|\eta\|_{L^{1}}-\frac{1}{3}\|\xi\|_{L^{1}}>0 \quad \text { or } \quad \hat{\eta}(k), \hat{\xi}(k) \in \mathbb{R}^{+} \text {for all } k \in \mathbb{Z}^{3}
$$

and

$$
\nabla \eta, \quad \nabla \xi \in L^{2}\left(\mathbb{T}^{3}\right)
$$

The main objective of the paper is to prove existence of a weak solution à la Leray for the steady compressible barotropic Navier-Stokes system with anisotropic and nonlocal diffusion. Anisotropic diffusion is present for instance in geophysical flows, see [22], while nonlocal diffusion is considered when studying confined fluids or in microfluidics where fluids flows thought narrow vessels. In order to achieve this goal, one key ingredient is the identity that we proposed in [2] which allowed us to give a simple proof for the existence of global weak-solutions for the anisotropic quasi-stationary Stokes system (compressible Brinkman equations).

More precisely, in this paper, we prove the following existence result

Theorem 1. Let us assume Hypothesis (H) be satisfied. There exists a constant $c_{0}$ such that if

$$
(1+|\theta|)|\theta| \mu \frac{2 \lambda+\mu}{(\lambda+\mu)^{2}} \leq c_{0}
$$

then there exists a pair $(\rho, u) \in L^{3(\gamma-1)}\left(\mathbb{T}^{3}\right) \times\left(W^{1, \frac{3(\gamma-1)}{\gamma}}\left(\mathbb{T}^{3}\right)\right)^{3}$ which is a weak-solution of the stationary Navier-Stokes system (1.4)-(1.5). 
Remark 1.1. Motivated by physically relevant phenomena like anisotropy or thermodynamically unstable pressure state laws, D. Bresch and P.E. Jabin introduced in [3] a new method for the identification of the pressure in the study of stability of solutions for the non-stationary compressible Navier-Stokes system. More precisely, if one considers a sequence of solutions generated by a sequence of initial data for which the corresponding sequence of initial densities is compact in $L^{1}$, then one is able to propagate this information for latter times via a nonlocal compactness criterion modulated with appropriate nonlinear weights. The idea in [3], propagation of compactness, is intimately related to the non-stationary transport equation and it does not seem to adapt to the stationary case.

Remark 1.2. The proof of Theorem 1 can be adapted to accommodate more general diffusion operators than (1.6). In particular, our method adapts to viscous stress tensors that include space-dependent coefficients or different convolution kernels for each component of $u$. In the opinion of the authors, the particular form of $\mathcal{A}$ proposed in (1.6), besides being physically relevant, see for instance [7] or [8], is also relatively easier to manipulate in computations.

To the authors's knowledge this is the first existence result of weak solutions taking in consideration anisotropic and nonlocal diffusion for the steady Navier-Stokes system. The first steps of the proof of Theorem 1 follow a rather well-known path: we consider an elliptic regularization for the system (1.4) to which classical theory can be applied and therefore we may construct a sequence of solutions parametrized by the regularization parameter. Of course, the more delicate part is to recover uniform estimates with respect to the regularization parameter and to show that the limiting object is a solution of the stationary Navier-Stokes system. The first key ingredient in the proof of stability is the new identity discovered by the authors in [2] in the context of the compressible Brinkman system. As it turns out, the $L^{2}$-integrability of the velocity field obtained via the basic energy estimate is not enough, better integrability is needed in order to justify rigorously the aforementioned identity. This is achieved by showing that it is possible to estimate the pressure $\rho^{\gamma}$ in a better space than $L^{2}$ : the smallness Condition (1.7) is required at this level. We point out that a similar condition is imposed in [3] in order to treat the non-stationary compressible Navier-Stokes system. In particular, one can consider an arbitrary anisotropic amplitude if the bulk viscosity is large enough.

The rest of the paper is organized in the following way: in Section 2, we recall existing results concerning weak solutions for the steady compressible Navier-Stokes equations and we discuss energy dissipation properties for the diffusion operator (1.6). In Section 3 we prove a nonlinear weak stability result, see Theorem 2 bellow, for the stationary compressible Navier-Stokes equation with anisotropic and nonlocal diffusion operator. In particular, we show that it is possible to recover strong convergence of the sequence of the gradients of the velocities and we show how to combine this fact with the compactness properties of the anisotropic viscous flux in order to identify the pressure. This is the main idea in the paper. In Section 4, we propose an approximate system and prove the existence of solutions to such system. Such approximate system is based on two layers of regularization: one ensuring ellipticity while the other one providing positivity of the density. In the last Section 5, we prove our main result, Theorem 1 first by establishing uniform estimates with respect to the two parameter and secondly using the non-linear stability results established in Section 3.

\section{Existing results for the steady Compressible Navier-Stokes system and energy dissipation properties for the diffusion operator $\mathcal{A}$}

Existing results. The problem of constructing solutions for the above system has been intensively studied and consequently there is a rather rich literature. We propose below a quick overview of the most recent results. First of all, we distinguish two types of solutions: strong respectively weaksolutions. Roughly speaking, a pair $(\rho, u)$ is a strong solution as soon as it verifies (1.1)-(1.2) almost everywhere on the domain of study, see the works of [1], [21], [27]. The existence theory of strong solutions always comes together with some "smallness condition" pertaining either to the size of the exterior forces $f, g$ acting on the system either to the size of some physical parameters like, for example, the Mach Number see [6]. However, one can prove that this solution is unique in some sense. 
A pair $(\rho, u)$ is weak-solution for (1.1) if it verifies this system in the sense of distributions and $\rho$ is just a Lebesgue function. One of the subtle points of the theory of weak-solutions comes from the genuine nonlinearity induced by the pressure term $p(\rho)=\rho^{\gamma}$ when $\gamma>1$. In order to make things clear we discuss briefly the most common strategy of constructing weak solutions, namely approximating system (1.1) with an elliptic system, typically by adding $\varepsilon \Delta \rho$ term in the mass equation. One expects that classical theory for elliptic equations to give rise to a sequence of solutions indexed by the approximation parameter $\varepsilon$. Of course, one should be able to obtain estimates verified by the sequence $\left(\rho^{\varepsilon}, u^{\varepsilon}\right)$ uniformly with respect to $\varepsilon$ and to show that the limit is a solution to the (1.1). However, we cannot reasonably expect to recover any regularity on $\rho^{\varepsilon}$, one is able only to recover that $\rho^{\varepsilon}$ is uniformly bounded in a Lebesgue space with integrability index greater than $\gamma$. Thus, as weak convergence is not commuting with nonlinear functions a delicate point is to be able to recover that the weak limit of the pressure sequence is the pressure associated to the limit density. This point proved to be difficult and the problem of existence of weak solutions resisted until 1998 when P.L. Lions in [16] proposed a solution combing two ingredients:

- renormalized transport theory which consists in the rigorous justification of the fact that $\rho$ also verifies

$$
\operatorname{div}(b(\rho) u)+\left(\rho b^{\prime}(\rho)-b(\rho)\right) \operatorname{div} u=0,
$$

for any $b$ sufficiently "well-behaved".

- the compactness properties of the so-called effective flux

$$
F=(2 \mu+\lambda) \operatorname{div} u-p(\rho)
$$

It is easy to give some rather informal hints why the above quantity behaves well: applying the divergence operator in the momentum equation of (1.1) we get that

$$
-\Delta F=\operatorname{div}(\rho f+g)-\operatorname{div}(\rho u \cdot \nabla u)
$$

and thus $\nabla F$ is of the same order as $\rho f+g-\rho u \cdot \nabla u$.

We note that the effective-flux, was used before in the context of the $1 D$ non-stationary NavierStokes system by J. Smoller and D. Hoff [14] and by D. Serre [26] when studying the problem of propagation of oscillations. In the multi-dimensional situation P.L. Lions used these two features in order to prove that

$$
\lim _{\varepsilon} b\left(\rho^{\varepsilon}\right)\left((2 \mu+\lambda) \operatorname{div} u^{\varepsilon}-p\left(\rho^{\varepsilon}\right)\right)=\lim _{\varepsilon} b\left(\rho^{\varepsilon}\right) \lim _{\varepsilon}\left((2 \mu+\lambda) \operatorname{div} u^{\varepsilon}-p\left(\rho^{\varepsilon}\right)\right),
$$

for any $b$ sufficiently "well-behaved" where $\left(\rho^{\varepsilon}, u^{\varepsilon}\right)$ is a sequence of solutions for the Navier-Stokes system. This identity is used in order to compare $\lim _{\varepsilon} \psi\left(\rho^{\varepsilon}\right)$ with $\psi\left(\lim _{\varepsilon} \rho^{\varepsilon}\right)$ for an appropriate convex function $\psi$ and to recover compactness for the density sequence. This nice argument, is of great generality: if $\gamma$ is large enough, it can be used to prove compactness for the density regardless of the domain where the problem of existence is studied and even for the non-stationary version of system (1.1). In [16], P.L. Lions constructed weak-solutions for system (1.1) if $\gamma>5 / 3$ in the case of finite domains with Dirichlet boundary condition for the velocity, in the whole space case $\mathbb{R}^{3}$, in the periodic boundary conditions and the case of an exterior domain. At this point it is worth mentioning that physical relevant values for the adiabatic coefficients include $\gamma=5 / 3$ for monatomic gases, $\gamma=7 / 5$ for diatomic gases, $\gamma=4 / 3$ for polyatomic gases. An argument leading to the relaxation of the condition $\gamma>5 / 3$ is due to S. Novo and A. Novotný [17] where the authors obtain existence of weak solutions for $\gamma>3 / 2$ and Dirichlet boundary conditions with potential body forces $f=\nabla h \in L^{\infty}$. It is worth mentioning that their argument relies in a crucial manner on E. Feireisl's work [9] on the non-stationary version of (1.1) where he introduced and studied a defect measure constructed with the help of truncations of the density.

The next improvement on the admissible bound on $\gamma$ came in the context of the periodic boundary conditions. More precisely, J. Březina and A. Novotný [5] constructed weak-solution for $\gamma>$ 
$(1+\sqrt{13}) / 3 \approx 1.53$ for volume non-potential body forces respectively for $\gamma>(3+\sqrt{41}) / 8 \approx 1.175$ in the case of potential body forces. Finally, the optimal result in the periodic framework, existence for $\gamma>1$ was obtained in [15] by S. Jiang, and C. Zhou. Concerning finite domains with Dirichlet boundary condition, the optimal result regarding the value of $\gamma$ is due to P. Plotnikov and W. Weigant [24] who constructed solutions for any $f \in L^{\infty}(\Omega), g=0$ with pressure functions $p(\rho)=\rho^{\gamma}$ for any $\gamma>1$, improving upon previous preliminary results obtained in [23] where the total mass condition (1.2) was replaced by $\int_{\Omega} \rho(x) d(x)^{-s} d x=M$ where $d(x)$ is the distance from $x$ to the boundary of the domain or [11] where the Dirichlet problem was solved for $\gamma>4 / 3$. We also mention results dealing with the relaxation of the conditions for the regularity of the boundary [18] or the case or non-compact boundaries [19]. The problem with the non-penetration condition $u \cdot n=0$ where $n$ is the unit normal at the boundary along with slip boundary conditions on the velocity was studied by M. Pokorný and P.B. Mucha in [25] where they are able to construct solutions with bounded density $\rho \in L^{\infty}$ in the case $\gamma>3$. More recently, E. Feireisl and A. Novotný [10] showed the existence of weak solutions for general inflow, outflow boundary conditions and monotone pressures that become singular near a finite value $\bar{\rho}$. For a survey on results obtained prior to the year 2003 one can consult the book of A. Novotný and I. Straškraba [20]. We emphasize that all the results obtained in the papers previously cited concern an isotropic linear viscous diffusion:

$$
\mathcal{A} u=\mu \Delta u+(\mu+\lambda) \nabla \operatorname{div} u .
$$

The objective of our paper is to enlarge the choice of the viscous stress tensors allowing more general diffusion operators of the form (1.6). Denoting

$$
\Delta_{\theta} \stackrel{\text { not. }}{=} \Delta+\theta \partial_{33}
$$

we can easily see that ${ }^{1}$

$$
\mathcal{A} u=\mu \Delta_{\theta} u+(\mu+\lambda) \nabla \operatorname{div} u+\eta * \Delta u+\xi * \nabla \operatorname{div} u .
$$

Energy dissipation for the new diffusion with anisotropic coefficients and nonlocal terms. Let us now discuss energy dissipation for the viscous operator that will be crucial to ensure existence of weak solution. In the following we use the notations

$$
\nabla_{\theta}=\left(\partial_{1}, \partial_{2},(1+\theta)^{\frac{1}{2}} \partial_{3}\right)
$$

and

$$
\operatorname{div}_{\theta} u=\partial_{1} u^{1}+\partial_{2} u^{2}+(1+\theta) \partial_{3} u^{3} .
$$

We can write ${ }^{2}$

$$
\begin{aligned}
\langle\mathcal{A} u, u\rangle= & \frac{1}{2} \mu \Delta_{\theta}\left(|u|^{2}\right)-\mu \nabla_{\theta} u: \nabla_{\theta} u \\
& +(\mu+\lambda) \operatorname{div}(u \operatorname{div} u)-(\mu+\lambda)(\operatorname{div} u)^{2} \\
& +\operatorname{div}(\eta * \nabla u u)-(\eta * \nabla u): \nabla u+\operatorname{div}(u \xi * \operatorname{div} u)-\xi * \operatorname{div} u \operatorname{div} u \\
= & \mathcal{B}(u, u)-\mathcal{C}(u, u)
\end{aligned}
$$

with

$$
\left\{\begin{aligned}
\mathcal{B}(u, u) \stackrel{\text { def. }}{=} & \frac{1}{2} \mu \Delta_{\theta}\left(|u|^{2}\right)+(\mu+\lambda) \operatorname{div}(u \operatorname{div} u) \\
& +\operatorname{div}(\eta * \nabla u u)-(\eta * \nabla u): \nabla u+\operatorname{div}((\xi * \operatorname{div} u) u)-(\xi * \operatorname{div} u) \operatorname{div} u \\
\mathcal{C}(u, u) \stackrel{\text { def. }}{=} & \mu \nabla_{\theta} u: \nabla_{\theta} u+(\mu+\lambda)(\operatorname{div} u)^{2} .
\end{aligned}\right.
$$

Let us observe that if

$$
u^{\varepsilon} \rightarrow u \text { strongly in }\left(L^{2}\left(\mathbb{T}^{3}\right)\right)^{3} \text { and } \nabla u^{\varepsilon} \rightarrow \nabla u \text { weakly in }\left(L^{2}\left(\mathbb{T}^{3}\right)\right)^{9}
$$

\footnotetext{
${ }^{1}$ We denote by $\eta * \Delta u$ respectively $\xi * \nabla \operatorname{div} u$ the $3 D$-vector fields with components $\left(\eta * \Delta u^{1}, \eta * \Delta u^{2}, \eta * \Delta u^{3}\right)$ respectively $\left(\xi * \partial_{1} \operatorname{div} u, \xi \partial_{2} \operatorname{div} u, \xi \partial_{3} \operatorname{div} u\right)$ where $u=\left(u^{1}, u^{2}, u^{3}\right)$.

${ }^{2}$ By $\nabla_{\theta} u$ we denote the $3 \times 3$ matrix whose $i^{t h}$ column is $\nabla_{\theta} u^{i}$. For two matrices $A, B$, we denote by $A: B=a_{i j} b_{i j}$.
} 
then

$$
\mathcal{B}\left(u^{\varepsilon}, u^{\varepsilon}\right) \rightarrow \mathcal{B}(u, u) \text { in the sense of distributions, }
$$

a fact that will prove crucial in our analysis. If the first condition on $\mathcal{A}$ in Hypothesis $(\mathrm{H})$ holds true then, we have that

$$
\begin{aligned}
-\int_{\mathbb{T}^{3}}\langle\mathcal{A} u, u\rangle & =\int_{\mathbb{T}^{3}}\left\{\mu \nabla_{\theta} u: \nabla_{\theta} u+(\mu+\lambda)(\operatorname{div} u)^{2}\right\}+\int_{\mathbb{T}^{3}}(\eta * \nabla u): \nabla u+\int_{\mathbb{T}^{3}} \xi * \operatorname{div} u \operatorname{div} u \\
& \geq\left(\min \{1,1+\theta\} \mu-\|\eta\|_{L^{1}}-\frac{1}{3}\|\xi\|_{L^{1}}\right) \int_{\mathbb{T}^{3}} \nabla u: \nabla u+(\mu+\lambda) \int_{\mathbb{T}^{3}}(\operatorname{div} u)^{2} .
\end{aligned}
$$

If the second condition on $\mathcal{A}$ in Hypothesis (H) holds true then, we have that

$$
\begin{aligned}
-\int_{\mathbb{T}^{3}}\langle\mathcal{A} u, u\rangle & =\int_{\mathbb{T}^{3}}\left\{\mu \nabla_{\theta} u: \nabla_{\theta} u+(\mu+\lambda)(\operatorname{div} u)^{2}\right\}+\int_{\mathbb{T}^{3}} \eta * \nabla u: \nabla u+\int_{\mathbb{T}^{3}} \xi * \operatorname{div} u \operatorname{div} u \\
& \geq \min \{1,1+\theta\} \mu \int_{\mathbb{T}^{3}} \nabla u: \nabla u+\sum_{k \in \mathbb{Z}^{3}} \sum_{i, j} \hat{\eta}(k)\left|\widehat{\partial_{j} u^{i}}(k)\right|^{2}+\sum_{k \in \mathbb{Z}^{3}} \hat{\xi}(k)|\widehat{\operatorname{div} u}(k)|^{2} .
\end{aligned}
$$

Remark finally that

$$
\operatorname{div} \mathcal{A} u=\left(\mu \Delta_{\theta}+(\mu+\lambda) \Delta\right) \operatorname{div} u+\Delta((\eta+\xi) * \operatorname{div} u)
$$

and

$$
\operatorname{div}_{\theta} \mathcal{A} u=\Delta_{\theta}\left(\mu \operatorname{div}_{\theta} u+(\mu+\lambda) \operatorname{div} u+\xi * \operatorname{div} u\right)+\Delta \eta * \operatorname{div}_{\theta} u
$$

\section{Nonlinear Weak stability}

This part of the paper concerns the nonlinear weak stability of the steady compressible Navier-Stokes system with anisotropic coefficients and nonlocal terms in the stress tensor. More precisely, we prove

Theorem 2. Let Hypothesis $(\boldsymbol{H})$ with an external force $g^{\varepsilon}$ be uniformly satisfied with respect to $\varepsilon$. Let $\left(\rho^{\varepsilon}, u^{\varepsilon}\right)_{\varepsilon>0}$ be a sequence of weak solutions of

$$
\left\{\begin{array}{l}
\operatorname{div}\left(\rho^{\varepsilon} u^{\varepsilon}\right)=0 \\
\operatorname{div}\left(\rho^{\varepsilon} u^{\varepsilon} \otimes u^{\varepsilon}\right)-\mathcal{A} u^{\varepsilon}+\nabla\left(\rho^{\varepsilon}\right)^{\gamma}=g^{\varepsilon} \\
\int_{\mathbb{T}^{3}} \rho^{\varepsilon}(x) d x=M, \quad \rho^{\varepsilon} \geq 0 \\
\int_{\mathbb{T}^{3}} u^{\varepsilon}(x) d x=0
\end{array}\right.
$$

satisfying

$$
\left\|\rho^{\varepsilon}\right\|_{L^{3(\gamma-1)\left(\mathbb{T}^{3}\right)}}+\left\|\nabla u^{\varepsilon}\right\|_{L}{\frac{3(\gamma-1)}{\gamma}\left(\mathbb{T}^{3}\right)} \leq C,
$$

where $C>0$ is a constant independent of $\varepsilon$. Then, there exists $(\rho, u) \in L^{3(\gamma-1)}\left(\mathbb{T}^{3}\right) \times\left(L^{\frac{3(\gamma-1)}{\gamma}}\right)^{3}\left(\mathbb{T}^{3}\right)$ such that up to a subsequence

$$
\left\{\begin{array}{l}
\rho^{\varepsilon} \rightarrow \rho \text { weakly in } L^{3(\gamma-1)}\left(\mathbb{T}^{3}\right), \\
\rho^{\varepsilon} \rightarrow \rho \text { in } L^{r}\left(\mathbb{T}^{3}\right) \text { for all } r \in[1,3(\gamma-1)), \\
u^{\varepsilon} \rightarrow u \text { in } L^{r}\left(\mathbb{T}^{3}\right) \text { for all } r \in[1,3(\gamma-1)), \\
\nabla u^{\varepsilon} \rightarrow \nabla u \text { weakly in }\left(L^{\frac{3(\gamma-1)}{\gamma}}\left(\mathbb{T}^{3}\right)\right)^{9}, \\
\nabla u^{\varepsilon} \rightarrow \nabla u \text { strongly in }\left(L^{r}\left(\mathbb{T}^{3}\right)\right)^{9} \text { for all } r \in\left[1, \frac{3(\gamma-1)}{\gamma}\right),
\end{array}\right.
$$

with $(\rho, u)$ a weak solution of the stationary compressible system (1.4)-(1.5).

The proof of Theorem 2 is rather non-standard in the context of problems coming from compressible fluid mechanics: we are able to prove that the sequence of velocity gradients converges strongly and 
recover a posteriori compactness properties of the equivalent anisotropic effective-flux. The main ingredient is the identity

$$
\operatorname{div}\left(\left(\overline{\rho^{\gamma}}-\rho^{\gamma}\right)^{\frac{1}{\gamma}}\right)+(\overline{\mathcal{C}(u, u)}-\mathcal{C}(u, u))\left(\overline{\rho^{\gamma}}-\rho^{\gamma}\right)^{\frac{1}{\gamma}-1}=0,
$$

where $\overline{\rho^{\gamma}}=\lim _{\varepsilon}\left(\rho^{\varepsilon}\right)^{\gamma}, \overline{\mathcal{C}(u, u)}=\lim _{\varepsilon} \mathcal{C}\left(u^{\varepsilon}, u^{\varepsilon}\right)$ where $\mathcal{C}(u, u)$ is defined in (2.5). As usually in PDEs, a stability property is the first important step before proving the existence of weak solutions. This is also the case in the present situation where it turns out that we can adapt the arguments used in Theorem 2 in order to obtain an existence result. This will be the subject of Section 5. Let us mention that the above identity is very sensitive to the specific form of the pressure as a power function. We are not able to treat the case of more general monotone pressure laws.

Proof of Theorem 2. Consider $\left(\rho^{\varepsilon}, u^{\varepsilon}\right)_{\varepsilon>0}$ a sequence verifying

$$
\left\{\begin{array}{l}
\operatorname{div}\left(\rho^{\varepsilon} u^{\varepsilon}\right)=0, \\
\operatorname{div}\left(\rho^{\varepsilon} u^{\varepsilon} \otimes u^{\varepsilon}\right)-\mathcal{A} u^{\varepsilon}+\nabla\left(\rho^{\varepsilon}\right)^{\gamma}=g^{\varepsilon}, \\
\int_{\mathbb{T}^{3}} \rho^{\varepsilon}(x) d x=M, \int_{\mathbb{T}^{3}} u^{\varepsilon}(x) d x=0, \quad \rho^{\varepsilon} \geq 0,
\end{array}\right.
$$

along with the following estimates

$$
\left\|\rho^{\varepsilon}\right\|_{L^{3(\gamma-1)\left(\mathbb{T}^{3}\right)}}+\left\|\nabla u^{\varepsilon}\right\|_{L^{\frac{3(\gamma-1)}{\gamma}\left(\mathbb{T}^{3}\right)}} \leq C
$$

where $C$ is independent of $\varepsilon$. Classical functional analysis results allow us to get the existence of functions $\left(\rho, u, \overline{\rho^{\gamma}}, \overline{\mathcal{C}(u, u)}\right)$ such that up to a subsequence

$$
\left\{\begin{array}{l}
\rho^{\varepsilon} \rightarrow \rho \text { weakly in } L^{3(\gamma-1)}\left(\mathbb{T}^{3}\right) \\
\left(\rho^{\varepsilon}\right)^{\gamma} \rightarrow \overline{\rho^{\gamma}} \text { weakly in } L^{\frac{3(\gamma-1)}{\gamma}}\left(\mathbb{T}^{3}\right) \\
\nabla u^{\varepsilon} \rightarrow \nabla u \text { weakly in } L^{\frac{3(\gamma-1)}{\gamma}}\left(\mathbb{T}^{3}\right) \\
\mathcal{C}\left(u^{\varepsilon}, u^{\varepsilon}\right) \rightarrow \overline{\mathcal{C}(u, u)} \text { weakly in } L^{\frac{3(\gamma-1)}{2 \gamma}}\left(\mathbb{T}^{3}\right) \\
u^{\varepsilon} \rightarrow u \text { strongly in } L^{q}\left(\mathbb{T}^{3}\right) \text { for any } 1 \leq q<3(\gamma-1) .
\end{array}\right.
$$

We deduce that

$$
\left\{\begin{array}{l}
\operatorname{div}(\rho u)=0, \\
\operatorname{div}(\rho u \otimes u)-\mathcal{A} u+\nabla \overline{\rho^{\gamma}}=g, \\
\int_{\mathbb{T}^{3}} \rho(x) d x=M, \int_{\mathbb{T}^{3}} u(x) d x=0, \rho^{\varepsilon} \geq 0 .
\end{array}\right.
$$

The more delicate problem is to be able to identify $\overline{\rho^{\gamma}}$ with $\rho^{\gamma}$. Let us explain the main ideas concerning the identification of the pressure in the isotropic case and then in the anisotropic case.

Identification of the pressure in the isotropic case

Let us briefly sketch the idea behind P.L. Lions's proof in the case when $\theta=\lambda=0$ and $\eta=\xi=0$, when the system reduces to

$$
\left\{\begin{array}{l}
\operatorname{div}\left(\rho^{\varepsilon} u^{\varepsilon}\right)=0 \\
\operatorname{div}\left(\rho^{\varepsilon} u^{\varepsilon} \otimes u^{\varepsilon}\right)-\mu \Delta u^{\varepsilon}+\nabla\left(\rho^{\varepsilon}\right)^{\gamma}=g^{\varepsilon} \\
\int_{\mathbb{T}^{3}} \rho^{\varepsilon}(x) d x=M, \int_{\mathbb{T}^{3}} u^{\varepsilon}(x) d x=0, \rho^{\varepsilon} \geq 0 .
\end{array}\right.
$$

As we allready mentioned in the introduction, there are two important points: first the regularity of the effective flux defined as

$$
F^{\varepsilon} \stackrel{\text { def. }}{=} \mu \operatorname{div} u^{\varepsilon}-\left(\rho^{\varepsilon}\right)^{\gamma}
$$

Indeed, applying the divergence operator in the momentum equation gives us

$$
-\Delta F^{\varepsilon}=-\operatorname{div}\left(\rho^{\varepsilon} u^{\varepsilon} \cdot \nabla u^{\varepsilon}\right)+\operatorname{div} g^{\varepsilon} .
$$


Thus $\left(\nabla F^{\varepsilon}\right)_{\varepsilon>0}$ is uniformly bounded in $W^{1, \frac{3(\gamma-1)}{2 \gamma-1}}\left(\mathbb{T}^{3}\right)$ and owing to the Rellich-Kondrachov theorem we obtain that

$$
b\left(\rho^{\varepsilon}\right) \cdot F^{\varepsilon} \rightarrow \overline{b(\rho)} \cdot F \text { weakly in } L^{1}\left(\mathbb{T}^{3}\right),
$$

for any continuous $b$ verifying some growth properties in 0 and at infinity where $b\left(\rho^{\varepsilon}\right) \rightarrow \overline{b(\rho)}$. The second part of the proof makes a clever use of the above identify. More precisely, fix a $\theta \in] 0,1[$. Owing to Proposition A.3 we get that

$$
\operatorname{div}\left(\left(\rho^{\varepsilon}\right)^{\theta} u^{\varepsilon}\right)+(\theta-1)\left(\rho^{\varepsilon}\right)^{\theta} \operatorname{div} u^{\varepsilon}=0
$$

which rewrites as

$$
\begin{aligned}
& \mu \operatorname{div}\left(\left(\rho^{\varepsilon}\right)^{\theta} u^{\varepsilon}\right)+(\theta-1)\left(\rho^{\varepsilon}\right)^{\theta}\left(\mu \operatorname{div} u^{\varepsilon}-\left(\rho^{\varepsilon}\right)^{\gamma}\right)+(\theta-1)\left(\rho^{\varepsilon}\right)^{\theta+\gamma} \\
& =\mu \operatorname{div}\left(\left(\rho^{\varepsilon}\right)^{\theta} u^{\varepsilon}\right)+(\theta-1)\left(\rho^{\varepsilon}\right)^{\theta} F^{\varepsilon}+(\theta-1)\left(\rho^{\varepsilon}\right)^{\theta+\gamma}=0
\end{aligned}
$$

such that passing to the limit yields

$$
\mu \operatorname{div}\left(\overline{\rho^{\theta}} u\right)+(\theta-1) \overline{\rho^{\theta}}\left(\mu \operatorname{div} u-\overline{\rho^{\gamma}}\right)+(\theta-1) \overline{\rho^{\theta+\gamma}}=0 .
$$

Using once more Proposition A.3 we get that

$$
\mu \operatorname{div}\left(\overline{\rho^{\theta}} \frac{1}{\theta} u\right)=\left(\frac{1}{\theta}-1\right)\left(\overline{\rho^{\theta+\gamma}}-\overline{\rho^{\theta}} \overline{\rho^{\gamma}}\right){\overline{\rho^{\theta}}}^{\frac{1}{\theta}-1}
$$

But by integration we get that

$$
\int_{\mathbb{T}^{3}}\left(\overline{\rho^{\theta+\gamma}}-\overline{\rho^{\theta}} \overline{\rho^{\gamma}}\right) \bar{\rho}^{\bar{\rho}^{\frac{1}{\theta}-1}}=0,
$$

which, by the positivity of the integrand implies that

$$
\overline{\rho^{\theta+\gamma}}-\overline{\rho^{\theta}} \overline{\rho^{\gamma}}=0,
$$

which implies by monotone operator theory that $\rho=\overline{\rho^{\gamma}} \frac{1}{\gamma}$.

Identification of the pressure in the anisotropic case.

The change of the algebraic structure of the effective flux in the anisotropic will make it impossible to adapt in a trivial manner the above approach. In order to highlight the differences with the isotropic case, in the following lines we continue our discussion for the case when $\theta>-1, \theta \neq 0, \lambda=0$ and $\eta=\xi=0$. There are two ways one can think of the anisotropic-effective flux. First, as explained in [3], we just take the divergence of the momentum equation and to write it as

$$
-\Delta_{\theta}\left(\mu \operatorname{div} u^{\varepsilon}-\left(\int_{\mathbb{T}^{3}}\left(\rho^{\varepsilon}\right)^{\gamma}+\Delta_{\theta}^{-1} \Delta\left(\left(\rho^{\varepsilon}\right)^{\gamma}-\int_{\mathbb{T}^{3}}\left(\rho^{\varepsilon}\right)^{\gamma}\right)\right)\right)=\operatorname{div} g^{\varepsilon}+\operatorname{div}\left(\rho^{\varepsilon} u^{\varepsilon} \cdot \nabla u^{\varepsilon}\right)
$$

and to try to mimic the proof in the isotropic case using

$$
F_{a n}^{\varepsilon}=\mu \operatorname{div} u^{\varepsilon}-\left(\int_{\mathbb{T}^{3}}\left(\rho^{\varepsilon}\right)^{\gamma}+\Delta_{\theta}^{-1} \Delta\left(\left(\rho^{\varepsilon}\right)^{\gamma}-\int_{\mathbb{T}^{3}}\left(\rho^{\varepsilon}\right)^{\gamma}\right)\right),
$$

as an effective flux (of course when $\theta=0, F_{a n}^{\varepsilon}$ coincides with $F^{\varepsilon}$ defined in (3.9)). This fails because we do not control the sign of

$$
\overline{\rho^{\theta}\left(\int_{\mathbb{T}^{3}} \rho^{\gamma}+\Delta_{\theta}^{-1} \Delta\left(\left(\rho^{\gamma}-\int_{\mathbb{T}^{3}} \rho^{\gamma}\right)\right)\right.}-\overline{\rho^{\theta}} \overline{\left(\int_{\mathbb{T}^{3}} \rho^{\gamma}+\Delta_{\theta}^{-1} \Delta\left(\left(\rho^{\gamma}-\int_{\mathbb{T}^{3}} \rho^{\gamma}\right)\right)\right.},
$$

as we do when $\theta=0$. Thus, in this case the equivalent of (3.10) is of no use for the identification of $\rho^{\gamma}$ with $\overline{\rho^{\gamma}}$. Secondly, we could apply $\operatorname{div}_{\theta}$, defined in (2.3), in the momentum equation in order to obtain

$$
-\Delta_{\theta}\left(\mu \operatorname{div}_{\theta} u^{\varepsilon}-\left(\rho^{\varepsilon}\right)^{\gamma}\right)=-\operatorname{div}_{\theta}\left(\rho^{\varepsilon} u^{\varepsilon} \cdot \nabla u^{\varepsilon}\right)+\operatorname{div}_{\theta} g^{\varepsilon},
$$


which yields compactness for the anisotropic effective-flux

$$
\tilde{F}_{a n}^{\varepsilon}=\mu \operatorname{div}_{\theta} u^{\varepsilon}-\left(\rho^{\varepsilon}\right)^{\gamma} .
$$

The problem is that this new quantity does not appear in the transport equation such that we cannot use it in order to replace $\overline{\rho^{\theta} \operatorname{div} u}$ with a more appropriate formula (unless, of course, we would have more information on $\partial_{3} u^{3}$ which is not the case). The key ingredient in the proof of Theorem 2 turns out to be the fact that we can recover compactness properties for the gradient of the velocity. In order to achieve this we have to use the renormalized stationary transport equation and to also take into account the momentum equation. More precisely the following proposition holds true:

Proposition 3.1. Under the hypothesis of Theorem 2, the following identity

$$
\frac{1}{\gamma-1} \operatorname{div}\left(u\left(\overline{\rho^{\gamma}}-\rho^{\gamma}\right)\right)+\left(\overline{\rho^{\gamma}}-\rho^{\gamma}\right) \operatorname{div} u+\overline{\mathcal{C}(u, u)}-\mathcal{C}(u, u)=0,
$$

holds true in the sense of distributions.

Proof. Owing to Proposition A.3 we get that

$$
\operatorname{div}\left(\left(\rho^{\varepsilon}\right)^{\gamma} u^{\varepsilon}\right)+(\gamma-1)\left(\rho^{\varepsilon}\right)^{\gamma} \operatorname{div} u^{\varepsilon}=0 .
$$

The fact that $\left(\rho^{\varepsilon}, u^{\varepsilon}\right)$ verify the bounds (3.5) allows us to extend the weak formulation of the velocity's equation to test functions $\psi$ for which $\psi \in\left(L^{2}\left(\mathbb{T}^{3}\right)\right)^{3}, \nabla \psi \in\left(L^{2}\left(\mathbb{T}^{3}\right)\right)^{9}$. Thus, taking $\varphi \in C^{\infty}\left(\mathbb{T}^{3}\right)$, may use $\varphi u$ as a test function in the weak formulation of the velocity's equation and using (2.4) and (3.14) we get that

$$
\operatorname{div}\left(\left(\rho^{\varepsilon}\right)^{\gamma} u^{\varepsilon}\right)=-\frac{(\gamma-1)}{\gamma}\left(\frac{1}{2} \operatorname{div}\left(\rho^{\varepsilon} u^{\varepsilon}\left|u^{\varepsilon}\right|^{2}\right)-\mathcal{B}\left(u^{\varepsilon}, u^{\varepsilon}\right)+\mathcal{C}\left(u^{\varepsilon}, u^{\varepsilon}\right)+u^{\varepsilon} g^{\varepsilon}\right)
$$

where $\mathcal{B}, \mathcal{C}$ are defined by (2.5). The convergence properties announced in (3.6) allow us to conclude that

$$
\operatorname{div}\left(\overline{\rho^{\gamma}} u\right)=-\frac{(\gamma-1)}{\gamma}\left(\frac{1}{2} \operatorname{div}\left(\rho u|u|^{2}\right)-\mathcal{B}(u, u)+\overline{\mathcal{C}(u, u)}+u g\right) .
$$

Of course, we can do the same manipulations to $(\rho, u)$ in order to obtain that

$$
\begin{aligned}
\operatorname{div}\left(\rho^{\gamma} u\right) & =\frac{(\gamma-1)}{\gamma}\left\{\operatorname{div}\left(\left(\overline{\rho^{\gamma}}-\rho^{\gamma}\right) u\right)-\left(\overline{\rho^{\gamma}}-\rho^{\gamma}\right) \operatorname{div} u\right\} \\
& -\frac{(\gamma-1)}{\gamma}\left(\frac{1}{2} \operatorname{div}\left(\rho u|u|^{2}\right)-\mathcal{B}(u, u)+\mathcal{C}(u, u)+u g\right) .
\end{aligned}
$$

Thus, by taking the difference we get (3.13) which ends the proof.

Next, we claim that

Proposition 3.2. Under the hypothesis of Theorem 2, we have that

$$
\nabla u^{\varepsilon} \rightarrow \nabla u \text { strongly in } L^{\frac{3(\gamma-1)}{\gamma}}\left(\mathbb{T}^{3}\right) .
$$

Proof. This will result from the manipulation of the identity proved in Proposition 3.1. Consider a regularizing kernel $\left(\omega_{\alpha}\right)_{\alpha>0}$ and using (3.13) we may write that

$$
\operatorname{div}\left(u \delta_{\alpha}\right)+(\gamma-1) \delta_{\alpha} \operatorname{div} u=-(\gamma-1) \omega_{\alpha} *(\overline{\mathcal{C}(u, u)}-\mathcal{C}(u, u))+r_{\alpha}\left(u, \rho, \overline{\rho^{\gamma}}\right)
$$

where

$$
\left\{\begin{array}{l}
\delta_{\alpha}=\omega_{\alpha} *\left(\overline{\rho^{\gamma}}-\rho^{\gamma}\right) \\
r_{\alpha}\left(u, \rho, \overline{\rho^{\gamma}}\right)=\operatorname{div}\left\{u \omega_{\alpha} *\left(\overline{\rho^{\gamma}}-\rho^{\gamma}\right)-\omega_{\alpha} *\left[\left(\overline{\rho^{\gamma}}-\rho^{\gamma}\right) u\right]\right\} \\
+(\gamma-1)\left\{\operatorname{div} u \omega_{\alpha} *\left(\overline{\rho^{\gamma}}-\rho^{\gamma}\right)-\omega_{\alpha} *\left[\left(\overline{\rho^{\gamma}}-\rho^{\gamma}\right) \operatorname{div} u\right]\right\}
\end{array}\right.
$$


Let $h>0$ be a constant and multiply the last equality with $\frac{1}{\gamma}\left(\delta_{\alpha}+h\right)^{\frac{1}{\gamma}-1}$ in order to get that

$$
\begin{aligned}
\operatorname{div}\left(u\left(\delta_{\alpha}+h\right)^{\frac{1}{\gamma}}\right) & =-\frac{\gamma-1}{\gamma}\left(\delta_{\alpha}+h\right)^{\frac{1}{\gamma}-1} \omega_{\alpha} *(\overline{\mathcal{C}(u, u)}-\mathcal{C}(u, u)) \\
& +\left(\delta_{\alpha}+h\right)^{\frac{1}{\gamma}-1} h \operatorname{div} u+\frac{1}{\gamma}\left(\delta_{\alpha}+h\right)^{\frac{1}{\gamma}-1} r_{\alpha}\left(u, \rho, \overline{\rho^{\gamma}}\right)
\end{aligned}
$$

Using Proposition A.2 we see that taking the limit $\alpha \rightarrow 0$ yields

$$
\begin{aligned}
\operatorname{div}\left(u\left(\left(\overline{\rho^{\gamma}}-\rho^{\gamma}\right)+h\right)^{\frac{1}{\gamma}}\right) & =-\frac{\gamma-1}{\gamma}(\overline{\mathcal{C}(u, u)}-\mathcal{C}(u, u))\left(\left(\overline{\rho^{\gamma}}-\rho^{\gamma}\right)+h\right)^{\frac{1}{\gamma}-1} \\
& +\left(\left(\overline{\rho^{\gamma}}-\rho^{\gamma}\right)+h\right)^{\frac{1}{\gamma}-1} h \operatorname{div} u .
\end{aligned}
$$

Integrating the last equation gives us

$$
\int_{\mathbb{T}^{3}}(\overline{\mathcal{C}(u, u)}-\mathcal{C}(u, u))\left(\left(\overline{\rho^{\gamma}}-\rho^{\gamma}\right)+h\right)^{\frac{1}{\gamma}-1}=\frac{\gamma h}{\gamma-1} \int_{\mathbb{T}^{3}}\left(\left(\overline{\rho^{\gamma}}-\rho^{\gamma}\right)+h\right)^{\frac{1}{\gamma}-1} \operatorname{div} u,
$$

which can be put under the form

$$
\begin{aligned}
& \int_{\left(\overline{\rho^{\gamma}}=\rho^{\gamma}\right)}(\overline{\mathcal{C}(u, u)}-\mathcal{C}(u, u))+\int_{\left(\overline{\left.\rho^{\gamma} \neq \rho^{\gamma}\right)}\right.}(\overline{\mathcal{C}(u, u)}-\mathcal{C}(u, u))\left(\frac{h}{\left(\overline{\rho^{\gamma}}-\rho^{\gamma}\right)+h}\right)^{1-\frac{1}{\gamma}} \\
& =\frac{\gamma h}{\gamma-1} \int_{\mathbb{T}^{3}}\left(\frac{h}{\left(\overline{\rho^{\gamma}}-\rho^{\gamma}\right)+h}\right)^{1-\frac{1}{\gamma}} \operatorname{div} u .
\end{aligned}
$$

Now, using that

$$
\left\{\begin{array}{l}
\lim _{h \rightarrow 0} \frac{h}{\left(\overline{\rho^{\gamma}}-\rho^{\gamma}\right)+h}=0 \text { a.e. on }\left(\overline{\rho^{\gamma}} \neq \rho^{\gamma}\right) \text { and } \\
\text { for any } h>0 \text { then } \frac{h}{\left(\overline{\rho^{\gamma}}-\rho^{\gamma}\right)+h} \leq 1 \text { a.e. on } \mathbb{T}^{3}
\end{array}\right.
$$

we get that

$$
\int_{\left(\overline{\rho^{\gamma}}=\rho^{\gamma}\right)}(\overline{\mathcal{C}(u, u)}-\mathcal{C}(u, u))=0
$$

As a consequence we get that

$$
(\overline{\mathcal{C}(u, u)}-\mathcal{C}(u, u)) \text { a.e. on }\left(\overline{\rho^{\gamma}}=\rho^{\gamma}\right)
$$

Then we see that (3.16) rewrites

$$
\int_{\left(\overline{\left.\rho^{\gamma} \neq \rho^{\gamma}\right)}\right.}(\overline{\mathcal{C}(u, u)}-\mathcal{C}(u, u))\left(\frac{h}{\left(\overline{\rho^{\gamma}}-\rho^{\gamma}\right)+h}\right)^{1-\frac{1}{\gamma}}=\frac{\gamma h}{\gamma-1} \int_{\mathbb{T}^{3}}\left(\frac{h}{\left(\overline{\rho^{\gamma}}-\rho^{\gamma}\right)+h}\right)^{1-\frac{1}{\gamma}} \operatorname{div} u,
$$

which we put under the form

$$
\int_{\left(\overline{\left.\rho^{\gamma} \neq \rho^{\gamma}\right)}\right.}(\overline{\mathcal{C}(u, u)}-\mathcal{C}(u, u))\left(\frac{1}{\left(\overline{\rho^{\gamma}}-\rho^{\gamma}\right)+h}\right)^{1-\frac{1}{\gamma}}=\frac{\gamma h^{\frac{1}{\gamma}}}{\gamma-1} \int_{\mathbb{T}^{3}}\left(\frac{h}{\left(\overline{\rho^{\gamma}}-\rho^{\gamma}\right)+h}\right)^{1-\frac{1}{\gamma}} \operatorname{div} u,
$$

such that using the inequality from (3.17) we get that

$$
\int_{\left(\overline{\rho^{\gamma} \neq \rho^{\gamma}}\right)}(\overline{\mathcal{C}(u, u)}-\mathcal{C}(u, u))\left(\frac{1}{\left(\overline{\rho^{\gamma}}-\rho^{\gamma}\right)+h}\right)^{1-\frac{1}{\gamma}} \leq \frac{\gamma h^{\frac{1}{\gamma}}}{\gamma-1}\|\operatorname{div} u\|_{L^{1}} .
$$

For all $n>0$ we have

$$
\left\{x: \overline{\rho^{\gamma}}(x) \geq \rho^{\gamma}(x)+1 / n\right\} \subset\left\{x: \overline{\rho^{\gamma}}(x) \neq \rho^{\gamma}(x)\right\}
$$


and as the integrand from the left hand side of the inequality (3.19) is positive, we get that

$$
\int_{\left(\overline{\rho^{\gamma}} \geq \rho^{\gamma}+1 / n\right)}(\overline{\mathcal{C}(u, u)}-\mathcal{C}(u, u))\left(\frac{1}{\left(\overline{\rho^{\gamma}}-\rho^{\gamma}\right)+h}\right)^{1-\frac{1}{\gamma}} \leq \frac{\gamma h^{\frac{1}{\gamma}}}{\gamma-1}\|\operatorname{div} u\|_{L^{1}} .
$$

Taking in account that

$$
\left\{\begin{array}{l}
\lim _{h \rightarrow 0} \frac{1}{\left(\overline{\rho^{\gamma}}-\rho^{\gamma}\right)+h}=\frac{1}{\left(\overline{\rho^{\gamma}}-\rho^{\gamma}\right)} \text { a.e. on }\left(\overline{\rho^{\gamma}} \geq \rho^{\gamma}+\frac{1}{n}\right) \text { and } \\
\left(\frac{1}{\left(\overline{\rho^{\gamma}}-\rho^{\gamma}\right)+h}\right)^{1-\frac{1}{\gamma}} \leq n^{1-\frac{1}{\gamma}} \text { a.e. on }\left(\overline{\rho^{\gamma}} \geq \rho^{\gamma}+\frac{1}{n}\right),
\end{array}\right.
$$

we get via the dominated convergence theorem that

$$
\int_{\left(\overline{\rho^{\gamma}} \geq \rho^{\gamma}+1 / n\right)}(\overline{\mathcal{C}(u, u)}-\mathcal{C}(u, u))\left(\overline{\rho^{\gamma}}-\rho^{\gamma}\right)^{\frac{1}{\gamma}-1}=0
$$

which yields

$$
\overline{\mathcal{C}(u, u)}-\mathcal{C}(u, u)=0 \text { a.e. on }\left\{x: \overline{\rho^{\gamma}}(x) \geq \rho^{\gamma}(x)+1 / n\right\} .
$$

As $n$ is arbitrary we deduce that

$$
\overline{\mathcal{C}(u, u)}=\mathcal{C}(u, u) \text { a.e. on }\left(\overline{\rho^{\gamma}}>\rho^{\gamma}\right) .
$$

Putting together the two relations (3.18) and (3.22) we get that

$$
\overline{\mathcal{C}(u, u)}-\mathcal{C}(u, u)=0 \text { a.e. on } \mathbb{T}^{3}
$$

and consequently

$$
\nabla u^{\varepsilon} \rightarrow \nabla u \text { in } L^{r}\left(\mathbb{T}^{3}\right),
$$

for all $r \in\left[1, \frac{3(\gamma-1)}{\gamma}\right)$. This concludes the proof of Proposition 3.2.

End of proof of Theorem 2. The fact that $\left(\nabla u^{\varepsilon}\right)_{\varepsilon>0}$ converges strongly to $\nabla u$ along with the fact that the anisotropic effective flux is compact will be used to identify $\overline{\rho^{\gamma}}$ with $\rho^{\gamma}$. Indeed, let us observe that owing to (2.10), when applying $\operatorname{div}_{\theta}$ in the second equation of (3.4) we obtain that

$$
-\Delta_{\theta}\left(\mu \operatorname{div}_{\theta} u^{\varepsilon}+(\mu+\lambda) \operatorname{div} u^{\varepsilon}+\xi * \operatorname{div} u^{\varepsilon}-\left(\rho^{\varepsilon}\right)^{\gamma}\right)=\Delta\left(\eta * \operatorname{div}_{\theta} u\right)-\operatorname{div}_{\theta}\left(\rho^{\varepsilon} u^{\varepsilon} \cdot \nabla u^{\varepsilon}\right)+\operatorname{div}_{\theta} g^{\varepsilon}
$$

such that

$$
\begin{aligned}
\nabla\left(\mu \operatorname{div}_{\theta} u^{\varepsilon}+(\mu+\lambda) \operatorname{div} u^{\varepsilon}+\xi * \operatorname{div} u^{\varepsilon}-\left(\rho^{\varepsilon}\right)^{\gamma}\right) & =-\left(-\Delta_{\theta}\right)^{-1}(-\Delta)\left(\eta * \operatorname{div}_{\theta} u\right) \\
& +\left(-\Delta_{\theta}\right)^{-1} \nabla\left(-\operatorname{div}_{\theta}\left(\rho^{\varepsilon} u^{\varepsilon} \cdot \nabla u^{\varepsilon}\right)+\operatorname{div}_{\theta} g^{\varepsilon}\right)
\end{aligned}
$$

and we recover that

$$
\mu \operatorname{div}_{\theta} u^{\varepsilon}+(\mu+\lambda) \operatorname{div} u^{\varepsilon}+\xi * \operatorname{div} u^{\varepsilon}-\left(\rho^{\varepsilon}\right)^{\gamma} \in W^{1, \frac{3(\gamma-1)}{2 \gamma-1}}\left(\mathbb{T}^{3}\right)
$$

and therefore, owing to the Rellich-Kondrachov we get that

$$
\lim _{\varepsilon \rightarrow 0}\left(\mu \operatorname{div}_{\theta} u^{\varepsilon}+(\mu+\lambda) \operatorname{div} u^{\varepsilon}+\xi * \operatorname{div} u^{\varepsilon}-\left(\rho^{\varepsilon}\right)^{\gamma}\right)=\mu \operatorname{div}_{\theta} u+(\mu+\lambda) \operatorname{div} u+\xi * \operatorname{div} u-\overline{\rho^{\gamma}}
$$

strongly in $L^{r}\left(\mathbb{T}^{3}\right)$ for all $r \in\left[1, \frac{3(\gamma-1)}{\gamma}\right)$. This implies that

$$
\begin{aligned}
& \lim _{\varepsilon \rightarrow 0} \rho^{\varepsilon}\left(\mu \operatorname{div}_{\theta} u^{\varepsilon}+(\mu+\lambda) \operatorname{div} u^{\varepsilon}+\xi * \operatorname{div} u^{\varepsilon}-\left(\rho^{\varepsilon}\right)^{\gamma}\right) \\
& =\rho\left(\mu \operatorname{div}_{\theta} u+(\mu+\lambda) \operatorname{div} u+\xi * \operatorname{div} u-\overline{\rho^{\gamma}}\right) \text { weakly in } L^{r}\left(\mathbb{T}^{3}\right),
\end{aligned}
$$

for some $r>1$. Of course, we may use the strong convergence of $\nabla u^{\varepsilon}$ to $\nabla u$ in order to conclude that

$$
\begin{aligned}
& \lim _{\varepsilon \rightarrow 0} \rho^{\varepsilon}\left(\mu \operatorname{div}_{\theta} u^{\varepsilon}+(\mu+\lambda) \operatorname{div} u^{\varepsilon}+\xi * \operatorname{div} u^{\varepsilon}\right) \\
& =\rho\left(\mu \operatorname{div}_{\theta} u+(\mu+\lambda) \operatorname{div} u+\xi * \operatorname{div} u\right) \text { weakly in } L^{r}\left(\mathbb{T}^{3}\right),
\end{aligned}
$$

for some $r>1$. Combining the last two identities we get that

$$
\lim _{\varepsilon \rightarrow 0}\left(\rho^{\varepsilon}\right)^{\gamma+1}=\rho \overline{\rho^{\gamma}} \text { weakly in } L^{r}\left(\mathbb{T}^{3}\right),
$$

with $r>1$ which, of course, implies that $\rho^{\gamma}=\overline{\rho^{\gamma}}$. This concludes the proof of Theorem 2 . 


\section{Construction of approximate solutions}

A weak solution for system (1.4)-(1.6) will be obtained as the limit of solutions of the following regularized system

$$
\left\{\begin{array}{l}
-\varepsilon \Delta \rho+\delta(\rho-M)+\operatorname{div}\left(\rho \omega_{\delta} * u\right)=0, \\
\frac{\delta}{2}\left(\rho u-\int_{\mathbb{T}^{3}} \rho u\right)+\operatorname{div}\left(\rho \omega_{\delta} * u \otimes u\right)-\mathcal{A} u+\nabla\left(\omega_{\delta} * \rho^{\gamma}\right)+\varepsilon\left(\nabla u \nabla \rho-\int_{\mathbb{T}^{3}} \nabla u \nabla \rho\right)=\omega_{\delta} * g, \\
\rho \geq 0, \int_{\mathbb{T}^{3}} \rho=M, \int_{\mathbb{T}^{3}} u=0,
\end{array}\right.
$$

when the regularization parameters $\delta, \varepsilon \in(0,1)^{2}$ tend to 0 . Above,

$$
\omega_{\delta}(\cdot)=\frac{1}{\delta^{3}} \omega\left(\frac{1}{\delta} \cdot\right)
$$

with $\omega \in \mathcal{D}\left(\mathbb{R}^{3}\right)$ a smooth, non-negative, even function which is compactly supported in the unit ball centered at the origin and with integral 1 . The fact that we can solve the above system is a consequence of the Leray-Schauder fixed point theorem, see Theorem A.1 from the Appendix. The objective of the next section is to construct solutions for (4.1).

\subsection{Existence of solutions for the approximate system (4.1)}

Let us fix $(\varepsilon, \delta) \in(0,1)^{2}$. We begin by the following proposition.

Proposition 4.1. Consider $v \in\left(W^{1, \infty}\left(\mathbb{T}^{3}\right)\right)^{3}$ and $M, \delta, \varepsilon>0$. Then there exists a unique positive solution $\rho \in W^{2,2}\left(\mathbb{T}^{3}\right)$ for the equation

$$
-\varepsilon \Delta \rho+\delta(\rho-M)+\operatorname{div}(\rho v)=0 .
$$

Moreover, there exists a positive constant $C(M, \varepsilon)$ depending on $\varepsilon$ and $M$ such that:

$$
\|\rho\|_{W^{2,2}} \leq C(M, \varepsilon)\left(1+\|v\|_{W^{1, \infty}}^{2}\right) .
$$

Proof. The proof is a classical application of the Leray-Schauder theorem. For any $r \in W^{1,2}\left(\mathbb{T}^{3}\right)$ we consider $T(r) \in W^{1,2}\left(\mathbb{T}^{3}\right)$ verifying

$$
-\varepsilon \Delta T(r)+\delta(T(r)-M)+\operatorname{div}(r v)=0 .
$$

The existence of $T(r, v) \in W^{1,2}\left(\mathbb{T}^{3}\right)$ is a consequence of the Lax-Milgram theorem. Continuity and compactness of the operator $T$. Observe that

$$
\int_{\mathbb{T}^{3}} T(r) d x=M
$$

Using (4.3) we have that

$$
\begin{aligned}
\varepsilon \int_{\mathbb{T}^{3}}|\nabla T(r)|^{2}+\delta \int_{\mathbb{T}^{3}}|T(r)|^{2} & \leq \delta M^{2}+\|r\|_{L^{6}}\|v\|_{L^{3}}\|\nabla T(r)\|_{L^{2}} \\
& \leq M^{2}+\frac{1}{2 \varepsilon}\|r\|_{L^{6}}^{2}\|v\|_{L^{\infty}}^{2}+\frac{\varepsilon}{2}\|\nabla T(r)\|_{L^{2}}^{2},
\end{aligned}
$$

which gives us

$$
\varepsilon^{\frac{1}{2}}\|\nabla T(r)\|_{L^{2}}+\delta^{\frac{1}{2}}\|T(r)\|_{L^{2}} \leq M+C(\varepsilon)\|v\|_{L^{\infty}}\|r\|_{W^{1,2}} .
$$

We also have that

$$
\begin{aligned}
\varepsilon\|\Delta T(r)\|_{L^{2}} & \leq \delta\|T(r)-M\|_{L^{2}}+\|r\|_{L^{2}}\|\operatorname{div} v\|_{L^{\infty}}+\|v\|_{L^{\infty}}\|\nabla r\|_{L^{2}} \\
& \leq C(M, \varepsilon)\left(1+\|v\|_{W^{1, \infty}}\|r\|_{W^{1,2}}\right)
\end{aligned}
$$


Consequently

$$
T(r) \in W^{2,2}\left(\mathbb{T}^{3}\right),
$$

such that using the Sobolev inequality, one also has that $\nabla T(r) \in L^{6}\left(\mathbb{T}^{3}\right)$ and $T(r) \in L^{r}\left(\mathbb{T}^{3}\right)$ for all $r \in[1, \infty]$ with

$$
\|\nabla T(r)\|_{L^{6}}+\|T(r)\|_{L^{r}} \leq C(M, \varepsilon)\left(1+\|v\|_{W^{1, \infty}}\|r\|_{W^{1,2}}\right) .
$$

Next, consider $r_{0} \in W^{1,2}\left(\mathbb{T}^{3}\right)$ and $r \in W^{1,2}\left(\mathbb{T}^{3}\right)$ such that

$$
\left\|r-r_{0}\right\|_{W^{1,2}} \leq 1
$$

First, we see that

$$
-\varepsilon \Delta\left(T(r)-T\left(r_{0}\right)\right)+\delta\left(T(r)-T\left(r_{0}\right)\right)+\operatorname{div}\left(\left(r-r_{0}\right) v\right)=0,
$$

such that multiplying with $T(r)-T\left(r_{0}\right)$ and applying Cauchy's inequality gives us

$$
\left\|T(r)-T\left(r_{0}\right)\right\|_{W^{1,2}} \leq C(M, \varepsilon)\|v\|_{L^{\infty}}\left\|r-r_{0}\right\|_{L^{2}} \leq C(M, \varepsilon)\|v\|_{L^{\infty}}\left\|r-r_{0}\right\|_{W^{1,2}} .
$$

Thus, $T$ is a continuous operator from $W^{1,2}\left(\mathbb{T}^{3}\right)$ into itself which is compact in view of (4.6). To apply the Leray-Schauder fixed point theorem, we define the set

$$
\mathcal{P}=\left\{\rho \in W^{1,2}\left(\mathbb{T}^{3}\right): \rho=\lambda T(\rho) \text { for some } \lambda \in(0,1]\right\} .
$$

and prove that it is a bounded set. The set $\mathcal{P}$ is bounded. Consider $\rho \in \mathcal{P}$ and $\lambda \in(0,1]$ such that

$$
-\varepsilon \Delta \rho+\delta(\rho-\lambda M)+\lambda \operatorname{div}(\rho v)=0 .
$$

We begin by proving that that such a $\rho$ is positive. In order to achieve this, consider

$$
\psi_{\eta}(s)=\frac{\sqrt{\eta+s^{2}}-s}{2}
$$

which is smooth and verifies for all $s \in \mathbb{R}$ and $\eta>0$

$$
\left\{\begin{array}{l}
0 \leq \psi_{\eta}(s)-\left(\frac{|s|-s}{2}\right) \leq \frac{\sqrt{\eta}}{2} \\
\psi_{\eta}^{\prime}(s) \leq 0, \psi_{\eta}^{\prime \prime}(s) \geq 0, \\
0 \leq \psi_{\eta}(s)-s \psi_{\eta}^{\prime}(s) \leq \frac{\sqrt{\eta}}{2}
\end{array}\right.
$$

Moreover, one can justify by regularization that for all $\eta>0$

$$
\begin{aligned}
& \delta\left(\rho \psi_{\eta}^{\prime}(\rho)-\lambda M \psi_{\eta}^{\prime}(\rho)\right)+\lambda \operatorname{div}\left(\psi_{\eta}(\rho) v\right)+\lambda\left(\rho \psi_{\eta}^{\prime}(\rho)-\psi_{\eta}(\rho)\right) \operatorname{div} v \\
& =\varepsilon \Delta \psi_{\eta}(\rho)-\varepsilon \psi_{\eta}^{\prime \prime}(\rho)|\nabla \rho|^{2} .
\end{aligned}
$$

We rewrite the last equation under the form

$$
\begin{aligned}
\delta \psi_{\eta}(\rho) & =\delta\left(\psi_{\eta}(\rho)-\rho \psi_{\eta}^{\prime}(\rho)\right)+\lambda M \delta \psi_{\eta}^{\prime}(\rho) \\
& -\lambda \operatorname{div}\left(\psi_{\eta}(\rho) v\right)-\lambda\left(\rho \psi_{\eta}^{\prime}(\rho)-\psi_{\eta}(\rho)\right) \operatorname{div} v \\
& +\varepsilon \Delta \psi_{\eta}(\rho)-\varepsilon \psi_{\eta}^{\prime \prime}(\rho)|\nabla \rho|^{2} .
\end{aligned}
$$

By integration and using (4.10) we end up with

$$
\delta \int_{\mathbb{T}^{3}} \psi_{\eta}(\rho) \leq \delta \int_{\mathbb{T}^{3}}\left(\psi_{\eta}(\rho)-\rho \psi_{\eta}^{\prime}(\rho)\right)-\lambda \int_{\mathbb{T}^{3}}\left(\rho \psi_{\eta}^{\prime}(\rho)-\psi_{\eta}(\rho)\right) \operatorname{div} v,
$$

which gives when $\eta \rightarrow 0$

$$
\frac{\delta}{2} \int_{\mathbb{T}^{3}}(|\rho|-\rho) \leq 0
$$


which implies that

$$
\rho(x) \geq 0 \text { a.e. on } \mathbb{T}^{3} .
$$

Next, we see that by integrating (4.9) we get that

$$
\|\rho\|_{L^{1}}=\int_{\mathbb{T}^{3}} \rho=\lambda M \leq M
$$

We also have that

$$
\begin{aligned}
\varepsilon \int_{\mathbb{T}^{3}}|\nabla \rho|^{2}+\delta \int_{\mathbb{T}^{3}} \rho^{2} & =\delta \lambda M^{2}+\lambda \int \rho^{2} \operatorname{div} v \leq \delta M^{2}+\lambda\|\operatorname{div} v\|_{L^{\infty}}\|\rho\|_{L^{2}}^{2} \\
& \leq \delta M^{2}+\|\operatorname{div} v\|_{L^{\infty}}\|\rho\|_{L^{2}}^{2} \leq \delta M^{2}+\|\operatorname{div} v\|_{L^{\infty}}\|\rho\|_{L^{1}}^{\frac{4}{5}}\|\rho\|_{L^{6}}^{\frac{12}{5}} \\
& \leq \delta M^{2}+\frac{1}{4 \alpha \varepsilon} M^{\frac{8}{5}}\|\operatorname{div} v\|_{L^{\infty}}^{2}+\alpha \varepsilon\|\rho-M\|_{L^{6}}^{2} \\
& \leq \delta M^{2}+\frac{1}{4 \alpha \varepsilon} M^{\frac{8}{5}}\|\operatorname{div} v\|_{L^{\infty}}^{2}+\alpha \varepsilon C\|\nabla \rho\|_{L^{2}}^{2} .
\end{aligned}
$$

We see that choosing $\alpha$ sufficiently small gives us

$$
\varepsilon \int_{\mathbb{T}^{3}}|\nabla \rho|^{2}+\delta \int_{\mathbb{T}^{3}} \rho^{2} \leq C(M, \varepsilon)\left(1+\|v\|_{W^{1, \infty}}^{2}\right)
$$

which means that the set is bounded.

Existence of solution to the nonlinear equation.

Thanks to the Leray-Schauder Theorem, see Theorem A.1, we get the existence of a fixed point for the operator $T$ defined by (4.2) which obviously, satisfies the equation

$$
-\varepsilon \Delta \rho+\delta(\rho-M)+\operatorname{div}(\rho v)=0 .
$$

and, moreover, verifies

$$
\left\{\begin{array}{l}
\int_{\mathbb{T}^{3}} \rho=M, \\
\varepsilon \int_{\mathbb{T}^{3}}|\nabla \rho|^{2}+\delta \int_{\mathbb{T}^{3}} \rho^{2} \leq C(M, \varepsilon)\left(1+\|v\|_{W^{1, \infty}}^{2}\right) .
\end{array}\right.
$$

We also have that

$$
\varepsilon\|\Delta \rho\|_{L^{2}} \leq \delta\|\rho-M\|_{L^{2}}+\|\operatorname{div}(\rho v)\|_{L^{2}} \leq\left(1+\|v\|_{W^{1, \infty}}\right)\|\rho\|_{W^{1,2}}
$$

which leads to

$$
\|\rho\|_{W^{2,2}} \leq C(M, \varepsilon)\left(1+\|v\|_{W^{1, \infty}}^{2}\right) .
$$

Let us consider two solutions $(\rho, \tilde{\rho})$ of $(4.11)$ and observe that their difference verifies

$$
-\varepsilon \Delta(\rho-\tilde{\rho})+\delta(\rho-\tilde{\rho})+\operatorname{div}((\rho-\tilde{\rho}) v)=0 .
$$

For all $\eta>0$, multiplying the above equation with $\varphi_{\eta}^{\prime}(\rho-\tilde{\rho})$ where

$$
\varphi_{\eta}(s)=\sqrt{\eta+s^{2}}
$$

integrating and making $\eta \rightarrow 0$ we get that

$$
\int_{\mathbb{T}^{3}}|\rho-\tilde{\rho}|=0
$$

The details are left as exercise for the reader. This ends the proof of Proposition 4.1. 
As was announced above, solutions for (4.1) are obtained as fixed points of an operator that is constructed in the following lines. We fix

$$
M>0, \quad g \in\left(L^{\frac{3(\gamma-1)}{2 \gamma-1}}\left(\mathbb{T}^{3}\right)\right)^{3} \text { with } \int_{\mathbb{T}^{3}} g=0,
$$

along with $(\varepsilon, \delta) \in(0,1)^{2}$, and for any $v \in\left(W^{1,2}\left(\mathbb{T}^{3}\right)\right)^{3}$ with $\int_{\mathbb{T}^{3}} v=0$ we consider $S(v) \in$ $\left(W^{1,2}\left(\mathbb{T}^{3}\right)\right)^{3}$ with $\int_{\mathbb{T}^{3}} S(v)=0$ verifying

$$
\begin{aligned}
-\mathcal{A} S(v) & =-\frac{\delta}{2}\left(\rho v-\int_{\mathbb{T}^{3}} \rho v\right)-\operatorname{div}\left(\rho \omega_{\delta} * v \otimes v\right)-\nabla\left(\omega_{\delta} * \rho^{\gamma}\right) \\
& -\varepsilon\left(\nabla v \nabla \rho-\int_{\mathbb{T}^{3}} \nabla v \nabla \rho\right)+\omega_{\delta} * g,
\end{aligned}
$$

where $\rho \in W^{2,2}\left(\mathbb{T}^{3}\right)$ is the unique solution of

$$
-\varepsilon \Delta \rho+\delta(\rho-M)+\operatorname{div}\left(\rho \omega_{\delta} * v\right)=0 .
$$

The existence of $S(v)$ is a consequence of the Lax-Milgram theorem applied in the closed subspace of $\left(W^{1,2}\left(\mathbb{T}^{3}\right)\right)^{3}$ of vector fields with zero mean. It remains now to prove that we have a fixed point to solve the nonlinear approximate system. This is the object of the following proposition.

Proposition 4.2. The operator $S$ defined by (4.13) admits a fixed point.

Proof. Proposition 4.2 is a consequence of the Schauder-Leray theorem (see Theorem A.1). We will first prove that $S$ is continuous and compact and in a second time that the set

$$
\mathcal{P}=\left\{u \in\left(W^{1,2}\left(\mathbb{T}^{3}\right)\right)^{3}: u=\lambda S(u) \text { for some } \lambda \in(0,1]\right\}
$$

is bounded. 1) Continuity and compactness of theoperator $S$. First, let us recall that

$$
-\varepsilon \Delta \rho+\delta(\rho-M)+\operatorname{div}\left(\rho \omega_{\delta} * v\right)=0,
$$

then

$$
\|\rho\|_{W^{2,2}} \leq C(M, \varepsilon, \delta)\left(1+\|v\|_{W^{1,2}}^{2}\right),
$$

see Proposition 4.1. In the following lines we show that $S(v)$ is actually more regular than $v$. We begin with

$$
\begin{aligned}
& \left\|\operatorname{div}\left(\rho \omega_{\delta} * v \otimes v\right)\right\|_{L^{\frac{3}{2}}} \\
& \leq\left\|\operatorname{div}\left(\rho \omega_{\delta} * v\right) v\right\|_{L^{\frac{3}{2}}}+\left\|\left(\rho \omega_{\delta} * v\right) \cdot \nabla v\right\|_{L^{\frac{3}{2}}} \\
& \leq\left\|\omega_{\delta} * v\right\|_{L^{\infty}}\|\nabla \rho\|_{L^{2}}\|v\|_{L^{6}}+\left\|\omega_{\delta} * \operatorname{div} v\right\|_{L^{\infty}}\|\rho\|_{L^{2}}\|v\|_{L^{6}}+\|\rho\|_{L^{6}}\left\|\omega_{\delta} * v\right\|_{L^{\infty}}\|\nabla v\|_{L^{2}} \\
& \leq C(M, \varepsilon, \delta)\left(1+\|v\|_{W^{1,2}}^{4}\right) .
\end{aligned}
$$

Using (4.15) we arrive at

$$
\left\|\omega_{\delta} * g\right\|_{L^{\infty}}+\left\|\nabla\left(\omega_{\delta} * \rho^{\gamma}\right)\right\|_{L^{\infty}} \leq C\left(M, \varepsilon, \delta,\|v\|_{W^{1,2}},\|g\|_{L^{\frac{6}{5}}}\right) .
$$

Using again (4.7) we have that

$$
\left\|\nabla v \nabla \rho-\int_{\mathbb{T}^{3}} \nabla v \nabla \rho\right\|_{L^{\frac{3}{2}}} \leq 2\|\nabla v\|_{L^{2}}\|\nabla \rho\|_{L^{6}} \leq 2\|\nabla v\|_{L^{2}}\|\rho\|_{W^{2,2}} \leq C(M, \varepsilon, \delta)\left(1+\|v\|_{W^{1,2}}^{3}\right) .
$$


Gathering the last three inequalities we get that

$$
\left\{\begin{array}{l}
\operatorname{div}\left(\rho \omega_{\delta} * v \otimes v\right)=\operatorname{div}\left(\rho \omega_{\delta} * v\right) v+\left(\rho \omega_{\delta} * v\right) \cdot \nabla v \in\left(L^{\frac{3}{2}}\left(\mathbb{T}^{3}\right)\right)^{3}, \\
\nabla\left(\omega_{\delta} * \rho^{\gamma}\right), \omega_{\delta} * g \in\left(L^{\infty}\left(\mathbb{T}^{3}\right)\right)^{3} \\
\varepsilon\left(\nabla v \nabla \rho-\int_{\mathbb{T}^{3}} \nabla v \nabla \rho\right), \frac{\delta}{2}\left(\rho v-\int_{\mathbb{T}^{3}} \rho v\right) \in\left(L^{\frac{3}{2}}\left(\mathbb{T}^{3}\right)\right)^{3}
\end{array}\right.
$$

which implies that

$$
\mathcal{A} S(v) \in\left(L^{\frac{3}{2}}\left(\mathbb{T}^{3}\right)\right)^{3}
$$

and consequently we get that

$$
S(v) \in\left(W^{2, \frac{3}{2}}\left(\mathbb{T}^{3}\right)\right)^{3} .
$$

Of course, sequences bounded in $W^{2, \frac{3}{2}}\left(\mathbb{T}^{3}\right)$ are precompact in $W^{1,2}\left(\mathbb{T}^{3}\right)$.

Consider $\left(v_{0}, v_{1}\right) \in\left(W^{1,2}\left(\mathbb{T}^{3}\right)\right)^{3} \times\left(W^{1,2}\left(\mathbb{T}^{3}\right)\right)^{3}$ such that

$$
\left\|v_{1}-v_{0}\right\|_{W^{1,2}} \leq 1
$$

Also, for $i \in\{0,1\}$ consider

$$
-\varepsilon \Delta \rho_{i}+\delta\left(\rho_{i}-M\right)+\operatorname{div}\left(\rho_{i} \omega_{\delta} * v_{i}\right)=0
$$

and

$$
\begin{aligned}
-\mathcal{A} S\left(v_{i}\right) & =-\frac{\delta}{2}\left(\rho_{i} v_{i}-\int_{\mathbb{T}^{3}} \rho_{i} v_{i}\right)-\operatorname{div}\left(\rho_{i} \omega_{\delta} * v_{i} \otimes v_{i}\right)-\nabla\left(\omega_{\delta} * \rho_{i}^{\gamma}\right) \\
& -\varepsilon\left(\nabla v_{i} \nabla \rho_{i}-\int \nabla v_{i} \nabla \rho_{i}\right)+\omega_{\delta} * g .
\end{aligned}
$$

First of all, the estimates (4.15) allow us to conclude that

$$
\left\|\rho_{0}\right\|_{W^{2,2}}+\left\|\rho_{1}\right\|_{W^{2,2}} \leq C(M, \varepsilon, \delta) .
$$

The difference $\left(\rho_{1}-\rho_{0}\right)$ verifies

$$
-\varepsilon \Delta\left(\rho_{1}-\rho_{0}\right)+\delta\left(\left(\rho_{1}-\rho_{0}\right)\right)+\operatorname{div}\left(\rho_{1} \omega_{\delta} * v_{1}-\rho_{0} \omega_{\delta} * v_{0}\right)=0,
$$

which provides the following estimate:

$$
\delta \int_{\mathbb{T}^{3}}\left|\rho_{1}-\rho_{0}\right| \leq \int_{\mathbb{T}^{3}}\left|\operatorname{div}\left(\rho_{0}\left(v_{1}-v_{0}\right)\right)\right| \leq\left\|\rho_{0}\right\|_{W^{1,2}}\left\|v_{1}-v_{0}\right\|_{W^{1,2}} .
$$

Next, we see that

$$
\begin{aligned}
\left\|\rho_{1}-\rho_{0}\right\|_{W^{1,2}} & \leq C\left\|\rho_{1} \omega_{\delta} * v_{1}-\rho_{0} \omega_{\delta} * v_{0}\right\|_{L^{2}} \\
& \leq C\left\|\omega_{\delta} * v_{1}\right\|_{L^{\infty}}\left\|\rho_{1}-\rho_{0}\right\|_{L^{2}}+C\left\|\rho_{0}\right\|_{L^{6}}\left\|\omega_{\delta} * v_{1}-\omega_{\delta} * v_{0}\right\|_{L^{3}} \\
& \leq C(M, \varepsilon, \delta)\left\|\rho_{1}-\rho_{0}\right\|_{L^{1}}^{\frac{2}{5}}+C(M, \varepsilon, \delta)\left\|v_{1}-v_{0}\right\|_{W^{1,2}} \\
& \leq C(M, \varepsilon, \delta)\left\|v_{1}-v_{0}\right\|_{W^{1,2}}^{\frac{2}{5}}+C(M, \varepsilon, \delta)\left\|v_{1}-v_{0}\right\|_{W^{1,2}} .
\end{aligned}
$$

Moreover, multiplying (4.18) with $-\Delta\left(\rho_{1}-\rho_{0}\right)$ one gets

$$
\begin{aligned}
& \varepsilon\left\|\Delta\left(\rho_{1}-\rho_{0}\right)\right\|_{L^{2}}^{2}+\delta\left\|\nabla\left(\rho_{1}-\rho_{0}\right)\right\|_{L^{2}}^{2} \\
& \leq\left\|\Delta\left(\rho_{1}-\rho_{0}\right)\right\|_{L^{2}}\left\|\operatorname{div}\left(\rho_{1} \omega_{\delta} * v_{1}-\rho_{0} \omega_{\delta} * v_{0}\right)\right\|_{L^{2}},
\end{aligned}
$$

from which we deduce that

$$
\left\|\Delta\left(\rho_{1}-\rho_{0}\right)\right\|_{L^{2}}+\left\|\nabla\left(\rho_{1}-\rho_{0}\right)\right\|_{L^{2}} \leq C(M, \varepsilon, \delta)\left(\left\|v_{1}-v_{0}\right\|_{W^{1,2}}^{\frac{2}{5}}+\left\|v_{1}-v_{0}\right\|_{W^{1,2}}\right)
$$


Next, by taking the difference of the velocity equations we end up with

$$
\begin{aligned}
\mathcal{A}\left(S\left(v_{1}\right)-S\left(v_{0}\right)\right) & =\frac{\delta}{2}\left(\rho_{0} v_{0}-\rho_{1} v_{1}\right)-\frac{\delta}{2}\left(\int_{\mathbb{T}^{3}} \rho_{0} v_{0}-\int_{\mathbb{T}^{3}} \rho_{1} v_{1}\right) \\
& +\operatorname{div}\left(\rho_{1} \omega_{\delta} * v_{1} \otimes v_{1}-\rho_{0} \omega_{\delta} * v_{0} \otimes v_{0}\right) \\
& +\nabla \omega_{\delta} *\left(\rho_{1}^{\gamma}-\rho_{0}^{\gamma}\right)+\varepsilon\left(\nabla v_{1} \nabla \rho_{1}-\nabla v_{0} \nabla \rho_{0}\right)-\varepsilon\left(\int_{\mathbb{T}^{3}} \nabla v_{1} \nabla \rho_{1}-\int_{\mathbb{T}^{3}} \nabla v_{0} \nabla \rho_{0}\right),
\end{aligned}
$$

from which we deduce that

$$
\begin{aligned}
\left\|S\left(v_{1}\right)-S\left(v_{0}\right)\right\|_{W^{1,2}} & \lesssim \delta\left\|\rho_{0} v_{0}-\rho_{1} v_{1}\right\|_{L^{\frac{6}{5}}}+\left\|\rho_{1} \omega_{\delta} * v_{1} \otimes v_{1}-\rho_{0} \omega_{\delta} * v_{0} \otimes v_{0}\right\|_{L^{2}} \\
& +\left\|\rho_{1}^{\gamma}-\rho_{0}^{\gamma}\right\|_{L^{2}}+2 \varepsilon\left\|\nabla v_{1} \nabla \rho_{1}-\nabla v_{0} \nabla \rho_{0}\right\|_{L^{\frac{6}{5}}} .
\end{aligned}
$$

Using (4.20), the first term is treated as follows

$$
\begin{aligned}
\left\|\rho_{0} v_{0}-\rho_{1} v_{1}\right\|_{L^{\frac{6}{5}}} & \leq\left\|v_{0}\right\|_{L^{3}}\left\|\left(\rho_{1}-\rho_{0}\right)\right\|_{L^{2}}+\left\|\rho_{1}\right\|_{L^{\frac{3}{2}}}\left\|v_{1}-v_{0}\right\|_{L^{6}} \\
& \leq C(M, \varepsilon, \delta)\left(\left\|v_{1}-v_{0}\right\|_{W^{1,2}}^{\frac{2}{5}}+\left\|v_{1}-v_{0}\right\|_{W^{1,2}}\right) .
\end{aligned}
$$

The second term is estimated as follows

$$
\begin{aligned}
& \left\|\rho_{1} \omega_{\delta} * v_{1} \otimes v_{1}-\rho_{0} \omega_{\delta} * v_{0} \otimes v_{0}\right\|_{L^{2}} \\
& \leq\left\|\omega_{\delta} * v_{1} \otimes v_{1}\right\|_{L^{3}}\left\|\rho_{1}-\rho_{0}\right\|_{L^{6}}+\left\|\rho_{0}\right\|_{L^{6}}\left\|\omega_{\delta} * v_{1} \otimes v_{1}-\omega_{\delta} * v_{0} \otimes v_{0}\right\|_{L^{3}} \\
& \leq\left\|v_{1}\right\|_{L^{6}}^{2}\left\|\rho_{1}-\rho_{0}\right\|_{W^{1,2}}+\left\|\rho_{0}\right\|_{L^{6}}\left\{\left\|v_{1}\right\|_{L^{6}}\left\|\omega_{\delta} * v_{1}-\omega_{\delta} * v_{0}\right\|_{L^{6}}+\left\|\omega_{\delta} * v_{0}\right\|_{L^{6}}\left\|v_{1}-v_{0}\right\|_{L^{6}}\right\} \\
& \leq C(M, \varepsilon, \delta)\left(\left\|v_{1}-v_{0}\right\|_{W^{1,2}}^{\frac{2}{5}}+\left\|v_{1}-v_{0}\right\|_{W^{1,2}}\right) .
\end{aligned}
$$

The third term is treated using the Sobolev inequality along with (4.15) and (4.21)

$$
\begin{aligned}
\left\|\rho_{1}^{\gamma}-\rho_{0}^{\gamma}\right\|_{L^{2}} & \leq\left(\left\|\rho_{1}\right\|_{L^{\infty}}^{\gamma-1}+\left\|\rho_{0}\right\|_{L^{\infty}}^{\gamma-1}\right)\left\|\rho_{1}-\rho_{0}\right\|_{L^{2}} \\
& \leq C\left(\left\|\rho_{1}\right\|_{W^{2,2}}^{\gamma-1}+\left\|\rho_{0}\right\|_{W^{2,2}}^{\gamma-1}\right)\left\|\rho_{1}-\rho_{0}\right\|_{W^{1,2}} \\
& \left.\leq C(M, \varepsilon, \delta)\left\|v_{1}-v_{0}\right\|_{W^{1,2}}^{\frac{2}{5}}+\left\|v_{1}-v_{0}\right\|_{W^{1,2}}\right) .
\end{aligned}
$$

The forth term is treated with the help of relations (4.15),(4.21) and (4.20)

$$
\begin{aligned}
& \left\|\nabla v_{1} \nabla \rho_{1}-\nabla v_{0} \nabla \rho_{0}\right\|_{L^{\frac{6}{5}}} \\
& \leq\left\|\nabla v_{1}\right\|_{L^{2}}\left\|\nabla\left(\rho_{1}-\rho_{0}\right)\right\|_{L^{3}}+\left\|\nabla \rho_{1}\right\|_{L^{3}}\left\|\nabla v-\nabla v_{0}\right\|_{L^{2}} \\
& \left.\leq C(M, \varepsilon, \delta)\left\|v_{1}-v_{0}\right\|_{W^{1,2}}^{\frac{2}{5}}+\left\|v_{1}-v_{0}\right\|_{W^{1,2}}\right) .
\end{aligned}
$$

From $(4.22),(4.23),(4.24),(4.25)$ we obtain that

$$
\left.\left\|S\left(v_{1}\right)-S\left(v_{0}\right)\right\|_{W^{1,2}} \leq C(M, \varepsilon, \delta)\left\|v_{1}-v_{0}\right\|_{W^{1,2}}^{\frac{2}{5}}+\left\|v_{1}-v_{0}\right\|_{W^{1,2}}\right) .
$$

Of course, the above relation shows that $S$ is continuos. Moreover, using (4.17) we get that the operator $S$ is compact. 2) The set $\mathcal{P}$ defined in (4.14) is bounded. In the following we prove that the set

$$
\left\{u \in\left(W^{1,2}\left(\mathbb{T}^{3}\right)\right)^{3}: u=\lambda S(u) \text { for some } \lambda \in(0,1]\right\}
$$

is bounded. Thus, consider $\lambda \in(0,1]$ and $u \in\left(W^{1,2}\left(\mathbb{T}^{3}\right)\right)^{3}$ such that $u=\lambda S(u)$. Obviously, one has

$$
\left\{\begin{array}{l}
-\varepsilon \Delta \rho+\delta(\rho-M)+\operatorname{div}\left(\rho \omega_{\delta} * u\right)=0, \\
\frac{\delta}{2}\left(\rho u-\int_{\mathbb{T}^{3}} \rho u\right)-\frac{1}{\lambda} \mathcal{A} u+\operatorname{div}\left(\rho \omega_{\delta} * u \otimes u\right)+\nabla\left(\omega_{\delta} * \rho^{\gamma}\right)+\varepsilon\left(\nabla u \nabla \rho-\int_{\mathbb{T}^{3}} \nabla u \nabla \rho\right)=\omega_{\delta} * g, \\
\int_{\mathbb{T}^{3}} \rho=M, \int_{\mathbb{T}^{3}} u=0 .
\end{array}\right.
$$


Observe that

$$
\begin{aligned}
& \left\langle\operatorname{div}\left(\rho \omega_{\delta} * u \otimes u\right)+\varepsilon \nabla u \nabla \rho, u\right\rangle \\
& =\frac{1}{2} \operatorname{div}\left(\rho \omega_{\delta} * u|u|^{2}\right)+\operatorname{div}\left(\rho \omega_{\delta} * u\right) \frac{|u|^{2}}{2}+\frac{\varepsilon}{2}\left\langle\nabla|u|^{2}, \nabla \rho\right\rangle \\
& =\frac{1}{2} \operatorname{div}\left(\rho \omega_{\delta} * u|u|^{2}\right)+(\varepsilon \Delta \rho-\delta(\rho-M)) \frac{|u|^{2}}{2}+\frac{\varepsilon}{2}\left\langle\nabla|u|^{2}, \nabla \rho\right\rangle \\
& =\frac{1}{2} \operatorname{div}\left(\rho \omega_{\delta} * u|u|^{2}\right)+\frac{\varepsilon}{2} \operatorname{div}\left(|u|^{2} \nabla \rho\right)-\delta(\rho-M) \frac{|u|^{2}}{2} .
\end{aligned}
$$

Next

$$
\int u \nabla\left(\omega_{\delta} * \rho^{\gamma}\right)=-\int \rho^{\gamma} \operatorname{div} \omega_{\delta} * u=\frac{4}{\gamma(\gamma-1)} \int_{\mathbb{T}^{3}}\left|\nabla \rho^{\frac{\gamma}{2}}\right|^{2}+\gamma \delta\left(\int_{\mathbb{T}^{3}} \rho^{\gamma}-M \int_{\mathbb{T}^{3}} \rho^{\gamma-1}\right) .
$$

Thus, we have that

$$
\begin{aligned}
& -\frac{1}{\lambda} \int_{\mathbb{T}^{3}}\langle\mathcal{A} u, u\rangle+\frac{\delta M}{2} \int_{\mathbb{T}^{3}}|u|^{2}+\frac{4 \varepsilon}{\gamma(\gamma-1)} \int_{\mathbb{T}^{3}}\left|\nabla \rho^{\frac{\gamma}{2}}\right|^{2}+\gamma \delta \int_{\mathbb{T}^{3}} \rho^{\gamma} \\
& =\int_{\mathbb{T}^{3}} \omega_{\delta} * g u+\gamma \delta M \int_{\mathbb{T}^{3}} \rho^{\gamma-1} .
\end{aligned}
$$

We use Young's inequality in order to obtain that

$$
\begin{aligned}
\gamma \delta M \int_{\mathbb{T}^{3}} \rho^{\gamma-1}+\int_{\mathbb{T}^{3}} \omega_{\delta} * g u & \leq \gamma \delta M\left(\int_{\mathbb{T}^{3}} \rho^{\gamma}\right)^{\frac{\gamma-1}{\gamma}}+\|g\|_{L^{\frac{6}{5}}}\|u\|_{L^{6}} \\
& \leq C(M, \gamma)\left(\|g\|_{L^{\frac{6}{5}}}^{2}+\delta\right)+\frac{1}{2 \lambda} \int_{\mathbb{T}^{3}}\langle\mathcal{A} u, u\rangle+\frac{\gamma \delta}{2} \int_{\mathbb{T}^{3}} \rho^{\gamma} .
\end{aligned}
$$

We obtain the existence of a constant $C(M, \gamma)$ depending only on $M$ and $\gamma$ such that

$$
\begin{aligned}
& -\frac{1}{2 \lambda} \int_{\mathbb{T}^{3}}\langle\mathcal{A} u, u\rangle+\frac{\delta M}{2} \int_{\mathbb{T}^{3}}|u|^{2}+\frac{4 \varepsilon}{\gamma(\gamma-1)} \int_{\mathbb{T}^{3}}\left|\nabla \rho^{\frac{\gamma}{2}}\right|^{2}+\frac{\gamma \delta}{2} \int_{\mathbb{T}^{3}} \rho^{\gamma} \\
& \leq C(M, \gamma)\left(\|g\|_{L^{\frac{6}{5}}}^{2}+\delta\right) \leq C(M, \gamma)\left(\|g\|_{L^{\frac{6}{5}}}^{2}+1\right) .
\end{aligned}
$$

The last estimate implies that $\mathcal{P}$ is a bounded set of $\left(W^{1,2}\left(\mathbb{T}^{3}\right)\right)^{3}$. Having proved that the operator $S$ verifies the hypothesis announced in Theorem A.1 we conclude that $S$ admits a fixed point. This concludes the proof of Proposition 4.2.

As an immediate consequence of Proposition 4.2 we get the following result

Corollary 3. Consider $(\varepsilon, \delta) \in(0,1)^{2}$. For all $M>0$ and $g \in\left(L^{\frac{3(\gamma-1)}{2 \gamma-1}}\left(\mathbb{T}^{3}\right)\right)^{3}$ with $\int_{\mathbb{T}^{3}} g=0$, there exists a solution $\left(\rho^{\varepsilon, \delta}, u^{\varepsilon, \delta}\right) \in W^{2,2}\left(\mathbb{T}^{3}\right) \times\left(W^{2, \frac{3}{2}}\left(\mathbb{T}^{3}\right)\right)^{3}$ of $(4.1)$ verifying the following estimates:

$$
\left\{\begin{array}{l}
-\frac{1}{2} \int_{\mathbb{T}^{3}}\left\langle\mathcal{A} u^{\varepsilon, \delta}, u^{\varepsilon, \delta}\right\rangle+\frac{4 \varepsilon}{\gamma(\gamma-1)} \int_{\mathbb{T}^{3}}\left|\nabla\left(\rho^{\varepsilon, \delta}\right)^{\frac{\gamma}{2}}\right|^{2}+\frac{\gamma \delta}{2} \int_{\mathbb{T}^{3}}\left(\rho^{\varepsilon, \delta}\right)^{\gamma} \leq C(M, \gamma)\left(\|g\|_{L^{\frac{6}{5}}}^{2}+1\right), \\
\left\|\Delta \rho^{\varepsilon, \delta}\right\|_{L^{\frac{3}{2}}}+\left\|\nabla \rho^{\varepsilon, \delta}\right\|_{L^{2}}^{2}+\left\|\mathcal{A} u^{\varepsilon, \delta}\right\|_{L^{\frac{6}{5}}} \leq C\left(M, \varepsilon,\|g\|_{L^{\frac{6}{5}}}\right)
\end{array}\right.
$$

Proof. The existence part of Corollary 3 follows by observing that a fixed point $u \in\left(W^{1,2}\left(\mathbb{T}^{3}\right)\right)^{3}$ of the operator $S$ defined by (4.13) turns out to verify (4.1). In order to finish the proof we must show that the pair $\left(\rho^{\varepsilon, \delta}, u^{\varepsilon, \delta}\right) \in W^{2,2}\left(\mathbb{T}^{3}\right) \times\left(W^{1, \frac{3}{2}}\left(\mathbb{T}^{3}\right)\right)^{3}$ constructed above verifies the announced estimates. We drop the $\varepsilon, \delta$ upper scripts in order to render the computations easier to follow. The first estimate 
of (4.32) is nothing else but (4.31) with $\lambda=1$. Of course, we will use it in order to prove the second inequality from (4.32). We begin with

$$
\begin{aligned}
\varepsilon\|\nabla \rho\|_{L^{2}}^{2}+\delta\|\rho\|_{L^{2}}^{2} & =\lambda\left(\delta M^{2}+\int_{\mathbb{T}^{3}} \omega_{\delta} * \operatorname{div} u \rho^{2}\right) \\
& \leq M^{2}+\|\operatorname{div} u\|_{L^{2}}\|\rho\|_{L^{4}}^{2} \leq M^{2}+C\left(1+\|g\|_{L^{\frac{6}{5}}}\right)\|\rho\|_{L^{1}}^{\frac{1}{5}}\|\rho\|_{L^{6}}^{\frac{9}{5}}
\end{aligned}
$$

which by means of the Young inequality yields

$$
\varepsilon\|\nabla \rho\|_{L^{2}}^{2}+\delta\|\rho\|_{L^{2}}^{2} \leq C\left(M, \varepsilon,\|g\|_{L^{\frac{6}{5}}}\right) .
$$

The estimate for the laplacian of $\rho$ is recovered using (4.33) and the Poincare inequality:

$$
\begin{aligned}
\varepsilon\|\Delta \rho\|_{L^{\frac{3}{2}}} & \leq \delta\|\rho-M\|_{L^{\frac{3}{2}}}+\left\|\rho \operatorname{div} \omega_{\delta} * u\right\|_{L^{\frac{3}{2}}}+\left\|\omega_{\delta} * u \nabla \rho\right\|_{L^{\frac{3}{2}}} \\
& \leq\|\nabla \rho\|_{L^{2}}+\|\rho\|_{L^{6}}\left\|\operatorname{div} \omega_{\delta} * u\right\|_{L^{2}}+\left\|\omega_{\delta} * u\right\|_{L^{6}}\|\nabla \rho\|_{L^{2}} \\
& \leq C\left(M, \varepsilon,\|g\|_{L^{\frac{6}{5}}}\right) .
\end{aligned}
$$

The last estimate along with Sobolev's inequality implies that

$$
\|\nabla \rho\|_{L^{3}} \leq C\left(M, \varepsilon,\|g\|_{L^{\frac{6}{5}}}\right) .
$$

In the following lines, we analyze the terms appearing in the velocity equation. Using (4.33) and (4.34) we obtain

$$
\|\rho u\|_{L^{3}}+\|\nabla u \nabla \rho\|_{L^{6 / 5}} \leq\|\rho\|_{L^{6}}\|u\|_{L^{6}}+\|\nabla u\|_{L^{2}}\|\nabla \rho\|_{L^{3}} \leq C\left(M, \varepsilon,\|g\|_{L^{\frac{6}{5}}}\right) .
$$

Next, writing that

$$
\begin{aligned}
\left\|\nabla\left(\omega_{\delta} * \rho^{\gamma}\right)\right\|_{L^{3 / 2}} & =\left\|\omega_{\delta} *\left(\rho^{\gamma / 2} \nabla \rho^{\gamma / 2}\right)\right\|_{L^{3 / 2}} \leq\left\|\rho^{\gamma / 2}\right\|_{L^{6}}\left\|\nabla \rho^{\gamma / 2}\right\|_{L^{2}} \\
& \leq C\left\|\nabla \rho^{\gamma / 2}\right\|_{L^{2}}^{2} \leq C\left(M, \varepsilon,\|g\|_{L^{\frac{6}{5}}}\right) .
\end{aligned}
$$

Notice that we also have that

$$
\begin{aligned}
\left\|\operatorname{div}\left(\rho \omega_{\delta} * u \otimes u\right)\right\|_{L^{\frac{6}{5}}} & =\left\|\left(\nabla \rho \cdot \omega_{\delta} * u\right) u\right\|_{L^{\frac{6}{5}}}+\left\|\rho \operatorname{div}\left(\omega_{\delta} * u\right) u\right\|_{L^{\frac{6}{5}}}+\left\|\rho \omega_{\delta} * u \cdot \nabla u\right\|_{L^{\frac{6}{5}}} \\
& \leq\|\nabla \rho\|_{L^{2}}\|u\|_{L^{6}}^{2}+\|\rho\|_{L^{6}}\|\operatorname{div} u\|_{L^{2}}\|u\|_{L^{6}}+\|\rho\|_{L^{6}}\|u\|_{L^{6}}\|\nabla u\|_{L^{2}} \\
& \leq C\left(M, \varepsilon,\|g\|_{L^{\frac{6}{5}}}\right)
\end{aligned}
$$

From (4.35), (4.36) and (4.37) it follows that $\mathcal{A} u \in\left(L^{6 / 5}\left(\mathbb{T}^{3}\right)\right)^{3}$ with

$$
\|\mathcal{A} u\|_{L^{\frac{6}{5}}} \leq C\left(M, \varepsilon,\|g\|_{L^{\frac{6}{5}}}\right) .
$$

This concludes the proof of Corollary 3 .

\section{Proof of Theorem 1}

To obtain Theorem 1 from the approximate system (4.1), it remains to pass to the limit first with respect to $\delta$ and secondly with respect to $\varepsilon$. As usually, in order to use the nonlinear weak stability obtained in a previous section, one important step will be to obtain estimates uniformly with respect to $\varepsilon$. 


\subsection{The approximate system in the limit $\delta \rightarrow 0$}

Owing to the Corollary 4.2 we see that for any $\varepsilon, \delta \in(0,1)$ we may consider $\left(\rho^{\varepsilon, \delta}, u^{\varepsilon, \delta}\right) \in W^{2, \frac{3}{2}}\left(\mathbb{T}^{3}\right) \times$ $\left(W^{2, \frac{3}{2}}\left(\mathbb{T}^{3}\right)\right)^{3}$ solution of (4.1) which verifies, uniformly in $\delta$ the estimates announced in (4.32). By virtue of the Rellich-Kondrachov theorem, these estimates are sufficient in order to pass to the limit when $\delta$ tends to 0 and obtain a solution of the limit system verifying the first estimate in (4.32). We skip the details. More precisely, we obtain the following:

Proposition 5.1. Consider $\varepsilon \in(0,1)$. For all $M>0$ and $g \in\left(L^{\frac{3(\gamma-1)}{2 \gamma-1}}\left(\mathbb{T}^{3}\right)\right)^{3}$ with $\int_{\mathbb{T}^{3}} g=0$, there exists $\left(\rho^{\varepsilon}, u^{\varepsilon}\right) \in W^{2, \frac{3}{2}}\left(\mathbb{T}^{3}\right) \times\left(W^{2, \frac{6}{5}}\left(\mathbb{T}^{3}\right)\right)^{3}$ verifying

$$
\left\{\begin{array}{l}
-\varepsilon \Delta \rho^{\varepsilon}+\operatorname{div}\left(\rho^{\varepsilon} u^{\varepsilon}\right)=0, \\
\operatorname{div}\left(\rho u^{\varepsilon} \otimes u^{\varepsilon}\right)-\mathcal{A} u^{\varepsilon}+\nabla\left(\rho^{\varepsilon}\right)^{\gamma}+\varepsilon\left(\nabla u^{\varepsilon} \nabla \rho^{\varepsilon}-\int_{\mathbb{T}^{3}} \nabla u^{\varepsilon} \nabla \rho^{\varepsilon}\right)=g, \\
\rho^{\varepsilon} \geq 0, \int_{\mathbb{T}^{3}} \rho^{\varepsilon}=M, \int_{\mathbb{T}^{3}} u^{\varepsilon}=0 .
\end{array}\right.
$$

along with the estimates

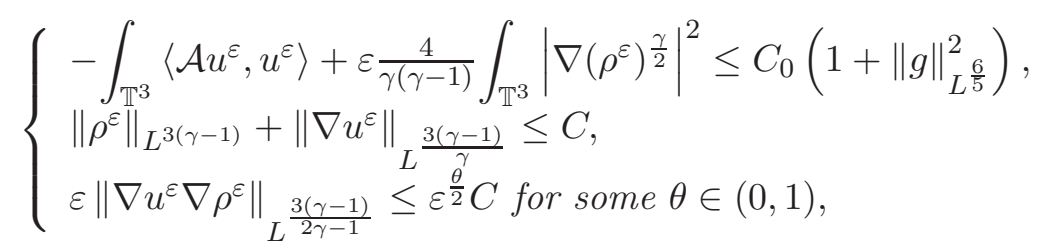

Where $C_{0}$ and $C=C\left(\theta, \mu, \lambda, \gamma,\|g\|_{L^{\frac{3(\gamma-1)}{2 \gamma-1}}},\|\eta\|_{L^{\frac{6(\gamma-1)}{4 \gamma-3}}},\|\xi\|_{L^{\frac{6(\gamma-1)}{4 \gamma-3}}}, M\right)$ are positive constants independent of $\varepsilon$.

We fill focus instead on proving the second and third estimates announced in (5.2) which say that it is possible to recover estimates for the density that are independent of $\varepsilon$ along with better integrability properties for the velocity $u$. This is the objective of the next section.

\subsubsection{Estimates for the density and improved estimates for the velocity}

We will drop the $\varepsilon$ superscript in order to ease the reading of the computation that follow. Thus, consider a pair $(\rho, u) \in W^{2, \frac{3}{2}}\left(\mathbb{T}^{3}\right) \times\left(W^{2, \frac{6}{5}}\left(\mathbb{T}^{3}\right)\right)^{3}$ solution of (5.1) verifying the first estimate from (5.2). Apply the divergence div operator in the momentum equation such as to obtain

$$
-\left\{\left(\mu \Delta_{\theta}+(\mu+\lambda) \Delta\right) \operatorname{div} u+\Delta((\eta+\xi) * \operatorname{div} u)\right\}+\Delta \rho^{\gamma}=\operatorname{div} g-\operatorname{div} \operatorname{div}(\rho u \otimes u)-\varepsilon \operatorname{div}(\nabla u \nabla \rho) .
$$

from which we obtain that

$$
\begin{aligned}
\rho^{\gamma} & =\int_{\mathbb{T}^{3}} \rho^{\gamma}+(2 \mu+\lambda) \operatorname{div} u+\left(I d-(2 \mu+\lambda)\left(\mu \Delta_{\theta}+(\mu+\lambda) \Delta\right)^{-1} \Delta\right)\left(\rho^{\gamma}-\int_{\mathbb{T}^{3}} \rho^{\gamma}\right) \\
& +(2 \mu+\lambda)\left(\mu \Delta_{\theta}+(\mu+\lambda) \Delta\right)^{-1}\{\Delta(\eta+\xi) * \operatorname{div} u+\operatorname{div} g-\operatorname{div} \operatorname{div}(\rho u \otimes u)-\varepsilon \operatorname{div}(\nabla u \nabla \rho)\} \\
& \stackrel{\text { not. }}{=} \sum_{i=1}^{7} T_{i} .
\end{aligned}
$$

In the following we will search for an $\alpha>0$ such that all $i \in \overline{1,7}$

$$
\int \rho^{\alpha} T_{i} \leq C(\|g\|, M)+\beta \int_{\mathbb{T}^{3}} \rho^{\alpha+\gamma},
$$

with a sufficiently small $\beta$ from which we will obtain an estimate of the form

$$
\int_{\mathbb{T}^{3}} \rho^{\alpha+\gamma} \leq C(\|g\|, M) .
$$


First term $T_{1}$ We simply write that

$$
\begin{aligned}
\int_{\mathbb{T}^{3}} \rho^{\alpha} \int_{\mathbb{T}^{3}} \rho^{\gamma} & \leq \frac{\alpha}{\alpha+\gamma}\left(\int_{\mathbb{T}^{3}} \rho^{\alpha}\right)^{\frac{\alpha+\gamma}{\alpha}}+\frac{\gamma}{\alpha+\gamma}\left(\int_{\mathbb{T}^{3}} \rho^{\gamma}\right)^{\frac{\alpha+\gamma}{\gamma}} \\
& \leq \frac{\alpha}{\alpha+\gamma}\left(\int_{\mathbb{T}^{3}} \rho\right)^{\left(1-\theta_{1}\right)(\alpha+\gamma)}\left(\int_{\mathbb{T}^{3}} \rho^{\alpha+\gamma}\right)^{\theta_{1}(\alpha+\gamma)} \\
& +\frac{\gamma}{\alpha+\gamma}\left(\int_{\mathbb{T}^{3}} \rho\right)^{\left(1-\theta_{2}\right)(\alpha+\gamma)}\left(\int_{\mathbb{T}^{3}} \rho^{\alpha+\gamma}\right)^{\theta_{2}(\alpha+\gamma)} \\
& \leq C(\alpha, \gamma, M, \beta)+\beta \int_{\mathbb{T}^{3}} \rho^{\alpha+\gamma}
\end{aligned}
$$

for any $\beta>0$ and some $\theta_{1}, \theta_{2} \in(0,1)$.

Second Term $T_{2}$. Using the equation of $\rho$ we see that

$$
-\varepsilon \Delta \rho^{\alpha}+\varepsilon \frac{4}{\alpha}\left|\nabla \rho^{\frac{\alpha}{2}}\right|^{2}+\operatorname{div}\left(\rho^{\alpha} u\right)+(\alpha-1) \rho^{\alpha} \operatorname{div} u=0,
$$

and consequently $T_{2}$ is a negative term:

$$
\int_{\mathbb{T}^{3}} \rho^{\alpha} T_{2}=\int_{\mathbb{T}^{3}} \rho^{\alpha} \operatorname{div} u=-\varepsilon \frac{4}{\alpha(\alpha-1)} \int_{\mathbb{T}^{3}}\left|\nabla \rho^{\frac{\alpha}{2}}\right|^{2} .
$$

Third term $T_{3}$. The third term is more delicate to treat because it is of the same order as $\rho^{\alpha+\gamma}$ such that we need the smallness assumption (1.7). Again using the mass equation we have that

$$
\begin{aligned}
& \int_{\mathbb{T}^{3}} \rho^{\alpha} T_{3} \\
& \leq\left\|\rho^{\alpha}\right\|_{L^{\frac{\alpha+\gamma}{\alpha}}}\left\|\left(I d-(2 \mu+\lambda)\left(\mu \Delta_{\theta}+(\mu+\lambda) \Delta\right)^{-1} \Delta\right)\left(\rho^{\gamma}-\int_{\mathbb{T}^{3}} \rho^{\gamma}\right)\right\|_{L^{\frac{\alpha+\gamma}{\gamma}}} \\
& \leq C(1+|\theta|) \mu|\theta| \frac{2 \lambda+\mu}{(\mu+\lambda)^{2}}\left\|\rho^{\alpha}\right\|_{L \frac{\alpha+\gamma}{\alpha}}\left\|\rho^{\gamma}\right\|_{L^{\frac{\alpha+\gamma}{\gamma}}}=C(1+|\theta|) \mu|\theta| \frac{2 \lambda+\mu}{(\mu+\lambda)^{2}} \int_{\mathbb{T}^{3}} \rho^{\alpha+\gamma},
\end{aligned}
$$

where we have used that the norm of the operator

$$
I d-(2 \mu+\lambda)\left(\mu \Delta_{\theta}+(\mu+\lambda) \Delta\right)^{-1} \Delta
$$

is controlled by

$$
C(1+|\theta|) \mu|\theta| \frac{2 \lambda+\mu}{(\mu+\lambda)^{2}},
$$

see Appendix after Theorem A.3. Consequently if this quantity is sufficiently small, we will be able to close the estimates absorbing this term by the left-hand side. This is satisfied for instance if $|\theta|$ is small enough or the bulk viscosity large enough.

Fourth term $T_{4}$. The fourth term is treated as follows

$$
\begin{aligned}
T_{4} & \leq C(\theta, \mu, \lambda)\|\rho\|_{L^{\alpha+\gamma}}^{\alpha}\|\eta+\xi\|_{L^{\frac{2(\alpha+\gamma)}{2 \gamma+\alpha}}}\|\operatorname{div} u\|_{L^{2}} \\
& \leq C(\theta, \mu, \lambda)\|\rho\|_{L^{\alpha+\gamma}}^{\alpha}\|\eta+\xi\|_{L^{\frac{2(\alpha+\gamma)}{2 \gamma+\alpha}}}\|g\|_{L^{\frac{6}{5}}}
\end{aligned}
$$

Fifth term $T_{5}$. The fifth term is treated as follows

$$
T_{5} \leq C(\theta, \mu, \lambda)\|\rho\|_{L^{\alpha+\gamma}}^{\alpha}\|g\|_{L^{\frac{3(\alpha+\gamma)}{4 \gamma+\alpha}}},
$$

provided that

$$
\frac{3(\alpha+\gamma)}{4 \gamma+\alpha}>1 \text { which yields } 2 \alpha>\gamma
$$


Sixth term $T_{6}$. The sixth term is treated as follows

$$
\begin{aligned}
T_{6} & \leq C(\theta, \mu, \lambda)\|\rho\|_{L^{\alpha+\gamma}}^{\alpha}\|\rho u \otimes u\|_{L^{\frac{\alpha+\gamma}{\gamma}}} \leq C(\theta, \mu, \lambda)\|\rho\|_{L^{\alpha+\gamma}}^{\alpha}\|u\|_{L^{6}}^{2}\|\rho\|_{L^{\frac{3(\alpha+\gamma)}{2 \gamma-\alpha}}} \\
& \leq C(\theta, \mu, \lambda)\|g\|_{L^{\frac{6}{5}}}^{2}\|\rho\|_{L^{\alpha+\gamma}}^{1+\alpha} .
\end{aligned}
$$

Of course in order to pass to the second line of (5.9) we need to have

$$
\frac{3(\alpha+\gamma)}{2 \gamma-\alpha} \leq \alpha+\gamma \text { which yields } \alpha \leq 2 \gamma-3
$$

This is the point where we see that a rather large adiabatic coefficient $\gamma$ is needed in order to recover that the pressure is a bit better than $L^{2}$.

Seventh term $T_{7}$. The seventh term is treated as follows. First we write that

$$
-\varepsilon \operatorname{div}(\nabla u \nabla \rho)=\operatorname{div}\left(\nabla u \Delta^{-1} \nabla \operatorname{div}(\rho u)\right) .
$$

Next, using the Sobolev inequality we get that

$$
\begin{aligned}
T_{7} & \leq\|\rho\|_{L^{\alpha+\gamma}}^{\alpha}\left\|\nabla u \Delta^{-1} \nabla \operatorname{div}(\rho u)\right\|_{L^{\frac{3(\alpha+\gamma)}{4 \gamma+\alpha}}}^{\alpha} \\
& \leq\|\rho\|_{L^{\alpha+\gamma}}^{\alpha}\|\nabla u\|_{L^{2}}\left\|\Delta^{-1} \nabla \operatorname{div}(\rho u)\right\|_{L^{\frac{6(\alpha+\gamma)}{5 \gamma-\alpha}}} \leq\|\rho\|_{L^{\alpha+\gamma}}^{\alpha}\|\nabla u\|_{L^{2}}\|\rho u\|_{L^{\frac{6(\alpha+\gamma)}{5 \gamma-\alpha}}} \\
& \leq\|\rho\|_{L^{\alpha+\gamma}}^{\alpha}\|\nabla u\|_{L^{2}}\|u\|_{L^{6}}\|\rho\|_{L^{\frac{3(\alpha+\gamma)}{2 \gamma-\alpha}}} \\
& \leq\|\rho\|_{L^{\alpha+\gamma}}^{1+\alpha}\|\nabla u\|_{L^{2}}^{2} .
\end{aligned}
$$

Conclusion. Finally, choosing $\alpha=2 \gamma-3$ and putting together all the above estimates concerning $T_{i}$ for $i=1, \cdots, 7$ we get that

$$
\|\rho\|_{L^{3(\gamma-1)}} \leq C\left(\theta, \mu, \lambda, \gamma,\|g\|_{L^{\frac{3(\gamma-1)}{2 \gamma-1}}},\|\eta\|_{L^{\frac{6(\gamma-1)}{4 \gamma-3}}},\|\xi\|_{L^{\frac{6(\gamma-1)}{4 \gamma-3}}}, M\right) .
$$

Of course, going back to the identity (5.3) and using the uniform bound on $\rho^{\gamma}$ in $L^{\frac{3(\gamma-1)}{\gamma}}$ and proceeding as we did in estimate (5.10) we can recover that

$$
\|\operatorname{div} u\|_{L^{\frac{3(\gamma-1)}{\gamma}}}+\|\rho\|_{L^{3(\gamma-1)}} \leq C\left(\theta, \mu, \lambda, \gamma,\|g\|_{L^{\frac{3(\gamma-1)}{2 \gamma-1}}},\|\eta\|_{L^{\frac{6(\gamma-1)}{4 \gamma-3}}},\|\xi\|_{L^{\frac{6(\gamma-1)}{4 \gamma-3}}}, M\right) .
$$

The last estimate can be used to get extra-integrability for the velocity field with respect to the basic energy estimate. This is achieved by observing that

$$
\begin{aligned}
\mu \nabla u & =\Delta_{\theta}^{-1} \nabla \operatorname{div}(\rho u \otimes u)+\Delta_{\theta}^{-1} \nabla^{2} \rho^{\gamma}--(\mu+\lambda) \Delta_{\theta}^{-1} \nabla^{2} \operatorname{div} u-\Delta_{\theta}^{-1} \nabla g \\
& -\Delta_{\theta}^{-1} \Delta(\eta * \nabla u)-\Delta_{\theta}^{-1} \nabla^{2}(\xi * \operatorname{div} u)+\varepsilon \Delta_{\theta}^{-1} \nabla(\nabla u \nabla \rho)
\end{aligned}
$$

such that we obtain

$$
\|\nabla u\|_{L} \frac{3(\gamma-1)}{\gamma} \leq C\left(\theta, \mu, \lambda, \gamma,\|g\|_{L^{\frac{3(\gamma-1)}{2 \gamma-1}}},\|\eta\|_{L^{\frac{6(\gamma-1)}{4 \gamma-3}}},\|\xi\|_{L^{\frac{6(\gamma-1)}{4 \gamma-3}}}, M\right) .
$$

Estimates for the gradient of the density. Finally, we aim at recovering some improved estimates for the gradient of $\rho$. In order to do that, we write in a first time that

$$
\varepsilon\|\nabla \rho\|_{L^{2}}^{2}=\int \rho^{2} \operatorname{div} u \leq\|\rho\|_{L^{4}}^{2}\|\operatorname{div} u\|_{L^{2}} \leq C\left(\theta, \mu, \lambda, \gamma,\|g\|_{L^{\frac{3(\gamma-1)}{2 \gamma-1}}},\|\eta\|_{\left.L^{\frac{6(\gamma-1)}{4 \gamma-3}},\|\xi\|_{L^{\frac{6(\gamma-1)}{4 \gamma-3}}}, M\right) .} .\right.
$$

Also, using

$$
\varepsilon \nabla \rho=\Delta^{-1} \nabla \operatorname{div}(\rho u)
$$


we get

$$
\varepsilon\|\nabla \rho\|_{L^{\frac{3(\gamma-1)}{2}}} \leq\|\rho\|_{L^{3(\gamma-1)}}\|u\|_{L^{3(\gamma-1)}} \leq C\left(\theta, \mu, \lambda, \gamma,\|g\|_{L^{\frac{3(\gamma-1)}{2 \gamma-1}}},\|\eta\|_{L^{\frac{6(\gamma-1)}{4 \gamma-3}}},\|\xi\|_{L^{\frac{6(\gamma-1)}{4 \gamma-3}}}, M\right) .
$$

Using (5.14) and (5.15) we obtain

$$
\varepsilon\|\nabla \rho\|_{L^{3}} \leq \varepsilon\|\nabla \rho\|_{L^{2}}^{\theta}\|\rho\|_{L^{\frac{3(\gamma-1)}{2}}}^{1-\theta} \leq \varepsilon^{\frac{\theta}{2}} C\left(\theta, \mu, \lambda, \gamma,\|g\|_{L^{\frac{3(\gamma-1)}{2 \gamma-1}}},\|\eta\|_{L^{\frac{6(\gamma-1)}{4 \gamma-3}}},\|\xi\|_{L^{\frac{6(\gamma-1)}{4 \gamma-3}}}, M\right),
$$

where $\theta \in(0,1)$ is given by

$$
\frac{1}{3}=\frac{\theta}{2}+\frac{2(1-\theta)}{3(\gamma-1)}
$$

Moreover,

$$
\begin{aligned}
\varepsilon\|\nabla u \nabla \rho\|_{L^{\frac{3(\gamma-1)}{2 \gamma-1}}} & \leq \varepsilon\|\nabla u\|_{L^{\frac{3(\gamma-1)}{\gamma}}}\|\nabla \rho\|_{L^{3}} \\
& \leq \varepsilon^{\frac{\theta}{2}} C\left(\theta, \mu, \lambda, \gamma,\|g\|_{L^{\frac{3(\gamma-1)}{2 \gamma-1}}},\|\eta\|_{L^{\frac{6(\gamma-1)}{4 \gamma-3}}},\|\xi\|_{\left.L^{\frac{6(\gamma-1)}{4 \gamma-3}}, M\right)}\right.
\end{aligned}
$$

\subsection{The limit passage $\varepsilon \rightarrow 0$.}

The Proof of Theorem 1 is based on the existence of solutions for the regularized system (5.1) and on an adoption of the proof of the stability result Theorem 1. Owing to Proposition 5.1, let us consider a sequence $\left(\rho^{\varepsilon}, u^{\varepsilon}\right)_{\varepsilon>0} \subset W^{2, \frac{3}{2}}\left(\mathbb{T}^{3}\right) \times\left(W^{2, \frac{6}{5}}\left(\mathbb{T}^{3}\right)\right)^{3}$ verifying (5.1) and uniformly in $\varepsilon$ the estimate (5.2). Using the theory of Sobolev spaces and the Rellich-Kondrachov theorem, we get the existence of functions $\left(\rho, u, \overline{\rho^{\gamma}}, \overline{\mathcal{C}(u, u)}\right)$ verifying

$$
\left\{\begin{array}{l}
\rho^{\varepsilon} \rightarrow \rho \text { weakly in } L^{3(\gamma-1)}\left(\mathbb{T}^{3}\right) \\
\left(\rho^{\varepsilon}\right)^{\gamma} \rightarrow \overline{\rho^{\gamma}} \text { weakly in } L^{\frac{3(\gamma-1)}{\gamma}}\left(\mathbb{T}^{3}\right) \\
\nabla u^{\varepsilon} \rightarrow \nabla u \text { weakly in } L^{\frac{3(\gamma-1)}{\gamma}}\left(\mathbb{T}^{3}\right) \\
\mathcal{C}\left(u^{\varepsilon}, u^{\varepsilon}\right) \rightarrow \overline{\mathcal{C}(u, u)} \text { weakly in } L^{\frac{3(\gamma-1)}{2 \gamma}}\left(\mathbb{T}^{3}\right) \\
u^{\varepsilon} \rightarrow u \text { strongly in } L^{q}\left(\mathbb{T}^{3}\right) \text { for any } 1 \leq q<3(\gamma-1) .
\end{array}\right.
$$

We recall that $\mathcal{C}$ is defined in relation $(2.5)$. We deduce that

$$
\left\{\begin{array}{l}
\operatorname{div}(\rho u)=0, \\
\operatorname{div}(\rho u \otimes u)-\mathcal{A} u+\nabla \overline{\rho^{\gamma}}=g, \\
\int_{\mathbb{T}^{3}} \rho(x) d x=M, \int_{\mathbb{T}^{3}} u(x) d x=0, \rho \geq 0 .
\end{array}\right.
$$

In order to identify $\overline{\rho^{\gamma}}$ with $\rho^{\gamma}$ we may proceed exactly as we did in Section 3 the only difference being that we have

$$
\frac{1}{\gamma-1} \operatorname{div}\left(u\left(\overline{\rho^{\gamma}}-\rho^{\gamma}\right)\right)+\left(\overline{\rho^{\gamma}}-\rho^{\gamma}\right) \operatorname{div} u+\overline{\mathcal{C}(u, u)}-C(u, u) \leq 0,
$$

instead of (3.13). Indeed, the negative sign comes from the fact that when we write the energy equations

$$
\begin{aligned}
& -\frac{\varepsilon}{\gamma-1} \Delta\left(\rho^{\varepsilon}\right)^{\gamma}+\frac{4 \varepsilon}{\gamma(\gamma-1)}\left|\nabla\left(\rho^{\varepsilon}\right)^{\frac{\gamma}{2}}\right|^{2}+\frac{\gamma}{\gamma-1} \operatorname{div}\left(\left(\rho^{\varepsilon}\right)^{\gamma} u^{\varepsilon}\right) \\
& =u^{\varepsilon} \nabla\left(\rho^{\varepsilon}\right)^{\gamma} \\
& =-u^{\varepsilon}\left\{\operatorname{div}\left(\rho u^{\varepsilon} \otimes u^{\varepsilon}\right)+\varepsilon \nabla u^{\varepsilon} \nabla \rho^{\varepsilon}\right\}+\left\langle\mathcal{A} u^{\varepsilon}, u^{\varepsilon}\right\rangle+g u^{\varepsilon}+\varepsilon u^{\varepsilon} \int_{\mathbb{T}^{3}} \nabla u^{\varepsilon} \nabla \rho^{\varepsilon} \\
& =-\frac{1}{2} \operatorname{div}\left(u^{\varepsilon} \rho^{\varepsilon}\left|u^{\varepsilon}\right|^{2}\right)-\frac{\varepsilon}{2} \operatorname{div}\left(u^{\varepsilon} \otimes \nabla \rho^{\varepsilon}\right)+\mathcal{B}\left(u^{\varepsilon}, u^{\varepsilon}\right)-\mathcal{C}\left(u^{\varepsilon}, u^{\varepsilon}\right)+g u^{\varepsilon}+\varepsilon u^{\varepsilon} \int_{\mathbb{T}^{3}} \nabla u^{\varepsilon} \nabla \rho^{\varepsilon} .
\end{aligned}
$$


Thus, using (2.6) and (5.16) we get that

$$
\frac{\gamma}{\gamma-1} \operatorname{div}\left(\overline{\rho^{\gamma}} u\right)=-\frac{1}{2} \operatorname{div}\left(u \rho|u|^{2}\right)+\mathcal{B}(u, u)-\overline{\mathcal{C}(u, u)}+g u-\Xi,
$$

where $\Xi$ is the limiting positive measure

$$
\Xi=\lim _{\varepsilon \rightarrow 0} \frac{4 \varepsilon}{\gamma(\gamma-1)}\left|\nabla\left(\rho^{\varepsilon}\right)^{\frac{\gamma}{2}}\right|^{2}
$$

But we also have that

$$
\begin{aligned}
\frac{\gamma}{\gamma-1} \operatorname{div}\left(\rho^{\gamma} u\right) & =\operatorname{div}\left(u\left(\overline{\rho^{\gamma}}-\rho^{\gamma}\right)\right)-\left(\overline{\rho^{\gamma}}-\rho^{\gamma}\right) \operatorname{div} u \\
& -\frac{1}{2} \operatorname{div}\left(u \rho|u|^{2}\right)+\mathcal{B}(u, u)-\mathcal{C}(u, u)+g u
\end{aligned}
$$

such that when taking the difference of (5.19) with (5.21) we end up with

$$
\operatorname{div}\left(u\left(\overline{\rho^{\gamma}}-\rho^{\gamma}\right)\right)+(\gamma-1)\left(\overline{\rho^{\gamma}}-\rho^{\gamma}\right) \operatorname{div} u+\overline{\mathcal{C}(u, u)}-\mathcal{C}(u, u)=-\Xi,
$$

with $\Xi$ the measure defined by (5.20). The proof of the fact that $\nabla u^{\varepsilon} \rightarrow \nabla u$ strongly in $L^{r}\left(\mathbb{T}^{3}\right)$ for all $r \in\left[1, \frac{3(\gamma-1)}{\gamma}\right)$ remains essentially the same as in Proposition 3.2. Observe that

$$
\begin{aligned}
\operatorname{div}\left(\rho^{\varepsilon} u^{\varepsilon} \otimes u^{\varepsilon}\right)+\varepsilon \nabla u^{\varepsilon} \nabla \rho^{\varepsilon} & =\operatorname{div}\left(\rho^{\varepsilon} u^{\varepsilon}\right) u^{\varepsilon}+\rho^{\varepsilon} u^{\varepsilon} \cdot \nabla u^{\varepsilon}+\varepsilon \nabla u^{\varepsilon} \nabla \rho^{\varepsilon} \\
& =\varepsilon \Delta \rho^{\varepsilon} u^{\varepsilon}+\varepsilon \nabla u^{\varepsilon} \nabla \rho^{\varepsilon}+\rho^{\varepsilon} u^{\varepsilon} \cdot \nabla u^{\varepsilon} \\
& =\varepsilon \operatorname{div}\left(u^{\varepsilon} \otimes \nabla \rho^{\varepsilon}\right)+\rho^{\varepsilon} u^{\varepsilon} \cdot \nabla u^{\varepsilon}
\end{aligned}
$$

Applying $\operatorname{div}_{\theta}$ in the velocity's equation we obtain that

$$
\begin{aligned}
\Delta_{\theta}\left(\mu \operatorname{div}_{\theta} u^{\varepsilon}+(\mu+\lambda) \operatorname{div} u^{\varepsilon}+\xi * \operatorname{div} u^{\varepsilon}-\left(\rho^{\varepsilon}\right)^{\gamma}\right) & =-\operatorname{div}\left(\nabla \eta * \operatorname{div}_{\theta} u^{\varepsilon}\right)-\varepsilon \operatorname{div}_{\theta} \operatorname{div}\left(u^{\varepsilon} \otimes \nabla \rho^{\varepsilon}\right) \\
& -\operatorname{div}_{\theta}\left(\rho^{\varepsilon} u^{\varepsilon} \cdot \nabla u^{\varepsilon}\right)-\operatorname{div}_{\theta} g
\end{aligned}
$$

thus, by denoting

$$
w^{\varepsilon} \stackrel{\text { not. }}{=} \mu \operatorname{div}_{\theta} u^{\varepsilon}+(\mu+\lambda) \operatorname{div} u^{\varepsilon}+\xi * \operatorname{div} u^{\varepsilon}-\left(\rho^{\varepsilon}\right)^{\gamma}+\varepsilon \Delta_{\theta}^{-1} \operatorname{div}_{\theta} \operatorname{div}\left(u^{\varepsilon} \otimes \nabla \rho^{\varepsilon}-\int_{\mathbb{T}^{3}} u^{\varepsilon} \otimes \nabla \rho^{\varepsilon}\right)
$$

using the uniform estimates (5.2) we get that

$$
w^{\varepsilon} \in W^{1, \frac{3(\gamma-1)}{2 \gamma-1}}\left(\mathbb{T}^{3}\right)
$$

such that using the Rellich-Kondrachov theorem we get that

$$
w^{\varepsilon} \rightarrow w=\mu \operatorname{div}_{\theta} u+(\mu+\lambda) \operatorname{div} u+\xi * \operatorname{div} u-\overline{\rho^{\gamma}}
$$

strongly for all $r \in\left[1, \frac{3(\gamma-1)}{\gamma}\right)$. Armed with this piece of information we proceed as in Section 3 concerning the nonlinear weak stability in order to conclude that $\rho^{\gamma}=\overline{\rho^{\gamma}}$. This ends the proof of Theorem 1.

\section{A Appendix}

\section{Functional analysis tools}

This section is devoted to a quick recall of the main results from functional analysis that we used thought the text. Consider $p \in[1, \infty), g \in L^{p}\left(\mathbb{T}^{3}\right)$ and $\omega \in \mathcal{D}\left(\mathbb{R}^{3}\right)$ a smooth, nonnegative, even function compactly supported in the unit ball centered at the origin and with integral equal to 1 . For all $\varepsilon>0$, we introduce the averaged functions

$$
g_{\varepsilon}=g * \omega_{\varepsilon}(x) \quad \text { where } \quad \omega_{\varepsilon}=\frac{1}{\varepsilon^{3}} \omega\left(\frac{x}{\varepsilon}\right) .
$$


We recall the following classical analysis result

$$
\lim _{\varepsilon \rightarrow 0}\left\|g_{\varepsilon}-g\right\|_{L^{p}\left(\mathbb{T}^{3}\right)}=0 .
$$

Moreover, for any multi-index $\alpha$ there exists a constant $C(\varepsilon, \alpha)$ such that

$$
\left\|\partial^{\alpha} g_{\varepsilon}\right\|_{L^{\infty}} \leq C(\varepsilon, \alpha)\|g\|_{L^{p}} .
$$

Next let us recall the following result concerning the commutator between the convolution with $\omega_{\varepsilon}$ and the product with a given function. More precisely, we have that

Proposition A.1 (Sobolev's inequality). Consider $p \in[1,3)$ and $g \in W^{1, p}\left(\mathbb{T}^{3}\right)$ with $\int_{\mathbb{T}^{3}} g=0$. Then,

$$
\|g\|_{L^{p_{\star}}} \leq\|\nabla g\|_{L^{p}}
$$

where $\frac{1}{p^{\star}}=\frac{1}{p}-\frac{1}{3}$.

Proposition A.2. Consider $\beta \in(1, \infty)$ and $(a, b)$ such that $a \in L^{\beta}\left(\mathbb{T}^{3}\right)$ and $b, \nabla b \in L^{p}\left(\mathbb{T}^{3}\right)$ where $\frac{1}{s}=\frac{1}{\beta}+\frac{1}{p} \leq 1$. Then, we have

$$
\lim r_{\varepsilon}(a, b)=0 \text { in } L^{s}\left(\mathbb{T}^{3}\right)
$$

where

$$
r_{\varepsilon}(a, b)=\partial_{i}\left(a_{\varepsilon} b\right)-\partial_{i}\left((a b)_{\varepsilon}\right)
$$

with $i \in\{1,2,3\}$.

One also has the following:

Proposition A.3. Consider $2 \leq \beta<\infty$ and $\lambda_{0}, \lambda_{1}$ such that $\lambda_{0}<1$ and $-1 \leq \lambda_{1} \leq \beta / 2-1$. Also, consider $\rho \in L^{\beta}\left(\mathbb{T}^{3}\right), \rho \geq 0$ a.e. and $u, \nabla u \in L^{2}\left(\mathbb{T}^{3}\right)$ verifying the following stationary transport equation

$$
\operatorname{div}(\rho u)=0
$$

in the sense of distributions. Then, for any function $b \in C^{0}([0, \infty)) \cap C^{1}((0, \infty))$ such that

$$
\left\{\begin{array}{l}
b^{\prime}(t) \leq c t^{-\lambda_{0}} \text { for } t \in(0,1] \\
\left|b^{\prime}(t)\right| \leq c t^{\lambda_{1}} \text { for } t \geq 1
\end{array}\right.
$$

it holds that

$$
\operatorname{div}(b(\rho) u)+\left\{\rho b^{\prime}(\rho)-b(\rho)\right\} \operatorname{div} u=0 .
$$

in the sense of distributions.

The proof of the above results follow by adapting in a straightforward manner lemmas 6.7. and 6.9 from the book of A. Novotný- I.Straškraba pages $155-188$. We end up this section with the following theorem that will be used to prove existence of solutions:

Theorem A.1 (Schauder-Leray). Let $\mathcal{T}$ be a continuous compact mapping of a Banach space $\mathcal{B}$ into itself with the property that there exists a real positive number $M>0$ such that

$$
\|x\|_{\mathcal{B}} \leq M
$$

for all $x$ such that $x=\lambda \mathcal{T} x$ for some $\lambda \in[0,1]$. Then $\mathcal{T}$ admits a fixed point.

For a proof of this result see Theorem 11.3. page 280 from [12]. 


\section{A.1 Fourier analysis tools}

In this section, we recall certain results concerning Fourier multiplier operators on the torus and the whole space and we recall the relation between them. More precisely, for the rest of the paper of this section we fix a bounded function $m: \mathbb{R}^{n} \backslash\{0\} \rightarrow \mathbb{C}$.

Definition A.1. We say that $m$ is a $(p, p)$-multiplier on $\mathbb{R}^{n}$ if the operator $S$ defined by

$$
S(g)=\mathcal{F}^{-1}(m(\xi) \mathcal{F}(g)),
$$

for all tempered distributions $g$ which have the support of their Fourier transform supported away from 0 can be extended to an operator that maps $L^{p}\left(\mathbb{R}^{n}\right)$ into itself. The class of all $(p, p)$-multipliers on $\mathbb{R}^{n}$ is denoted $M_{p}\left(\mathbb{R}^{n}\right)$ and we define the $M_{p}$-norm of $m$ as being the operatorial norm of the associated operator $S$ i.e.

$$
\|m\|_{M_{p}\left(\mathbb{R}^{n}\right)}: \stackrel{\text { def. }}{=}\|S\|_{\mathcal{L}\left(L^{p}\left(\mathbb{R}^{n}\right), L^{p}\left(\mathbb{R}^{n}\right)\right)} .
$$

In the following we denote $L_{0}^{p}\left(\mathbb{T}^{n}\right)$ the closed subspace of $L^{p}\left(\mathbb{T}^{n}\right)$ with mean value 0 .

Definition A.2. We say that $\{m(k)\}_{k \in \mathbb{Z}^{n} \backslash\{0\}}$ is a $(p, p)$-multiplier on the torus if the operator $T$ defined by

$$
T(P)(x)=P(x)=\sum_{k \in \mathbb{Z}^{n} \backslash\{0\}} m(k) a_{k} \exp (2 \pi i k \cdot x),
$$

for all trigonometric polynomials with zero mean i.e.

$$
P(x)=\sum_{k \in \mathbb{Z}^{n}} a_{k} \exp (2 \pi i k \cdot x)
$$

with $\left(a_{k}\right)_{k \in \mathbb{Z}^{n}}$ with finite support and $a_{0}=0$, can be extended to an operator that maps $L_{0}^{p}\left(\mathbb{T}^{n}\right)$ into itself. The class of all $(p, p)$-multipliers on the torus is denoted $M_{p}\left(\mathbb{Z}^{n}\right)$ and we define the $M_{p}$-norm of $m$ as being the operatorial norm of the associated operator $T$ i.e.

$$
\|m\|_{M_{p}\left(\mathbb{Z}^{n}\right)}: \stackrel{\text { def. }}{=}\|S\|_{\mathcal{L}\left(L^{p}\left(\mathbb{T}^{n}\right), L^{p}\left(\mathbb{T}^{n}\right)\right)} .
$$

One of the classical subjects in Fourier analysis tries to capture the properties that $m$ has to satisfy in order to be a $(p, p)$-Fourier multiplier. In the following, we recall Mihlin's multiplier theorem that gives a sufficient conditions such that $m$ to be a Fourier multiplier on $\mathbb{R}^{n}$.

Theorem A.2. Let $m(\xi)$ be a complex-valued bounded function on $\mathbb{R}^{n} \backslash\{0\}$ that satisfies Mihlin's condition

$$
\left|\partial_{\xi}^{\alpha} m(\xi)\right| \leq A|\xi|^{-|\alpha|},
$$

for all multi-indices $|\alpha| \leq\left[\frac{n}{2}\right]+1$. Then, for all $p \in(1, \infty)$, $m$ is a $(p, p)$-multiplier on $\mathbb{R}^{n}$ and there exists a constant $C_{n}$ depending only on the dimension $n$ such that for all $g \in L^{p}\left(\mathbb{R}^{n}\right)$ :

$$
\|m\|_{M_{p}\left(\mathbb{R}^{n}\right)} \leq C_{n} \max \left\{p, \frac{1}{p-1}\right\}\left(A+\|m\|_{L^{\infty}\left(\mathbb{R}^{n}\right)}\right)\|g\|_{L^{p}\left(\mathbb{R}^{n}\right)} .
$$

A proof of this result can be found in L. Grafakos's book, see [13] Theorem 5.2.7., page 367.

Remark A.1. One can check by direct calculation that $m: \mathbb{R}^{3} \backslash\{0\} \rightarrow \mathbb{R}$ defined by

$$
m(\xi)=\frac{\left|\xi_{3}\right|^{2}}{a_{1}\left|\xi_{1}\right|^{2}+a_{2}\left|\xi_{2}\right|^{2}+a_{3}\left|\xi_{3}\right|^{2}},
$$

verifies the Milhin condition (A.6) with $A=\max \left\{A_{0}, A_{1}, A_{2}\right\}$ with

$$
A_{0}=\frac{1}{a_{3}}
$$


and

and

$$
A_{1}=\max \left\{\frac{\sqrt{a_{1}}}{a_{3}}, \frac{\sqrt{a_{2}}}{a_{3}}, \frac{1}{\sqrt{a_{3}}}\right\} \frac{1}{\sqrt{\min \left\{a_{1}, a_{2}, a_{3}\right\}}},
$$

$$
A_{2}=\max \left\{\frac{a_{1}}{a_{3}}, \frac{a_{2}}{a_{3}}, 1\right\} \frac{1}{\min \left\{a_{1}, a_{2}, a_{3}\right\}},
$$

where each $A_{i}$ represents the constant appearing in the (A.6) respectively for the $|\alpha|=0,|\alpha|=1$ and $|\alpha|=2$ derivatives. Milhin's theorem implies that $m$ is a Fourier multiplier on $\mathbb{R}^{n}$.

Definition A.3. Let $\xi_{0} \in \mathbb{R}^{n}$. A bounded function $m$ on $\mathbb{R}^{n}$ is called regulated at the point $\xi_{0}$ if

$$
\lim _{\varepsilon \rightarrow 0} \int_{|t| \leq \varepsilon}\left(m\left(\xi_{0}-\xi\right)-m\left(\xi_{0}\right)\right) d \xi=0 .
$$

Obviously, if $m$ is continuous in $\xi_{0}$ then $m$ is regulated at the point $\xi_{0}$. The following result is the key point in transferring the Milhin theorem on the torus:

Lemma A.1. Let $T$ be a operator on $\mathbb{R}^{n}$ whose multiplier is $m(\xi)$ and let $S$ be the operator on $\mathbb{T}^{n}$ whose multiplier is the sequence $\{m(k)\}_{k \in \mathbb{Z}^{n}}$. Assume that $m(\xi)$ is regular at every point in $\mathbb{Z}^{n} \backslash\{0\}$. Suppose that $P$ and $Q$ are trigonometric polynomials on $\mathbb{T}^{n}$ and let $L_{\varepsilon}(x)=\exp \left(-\pi \varepsilon|x|^{2}\right)$ for $x \in \mathbb{R}^{n}$ and $\varepsilon>0$. Then the following identity is valid whenever $\alpha, \beta>0$ and $\alpha+\beta=1$ :

$$
\lim _{\varepsilon \rightarrow 0} \varepsilon^{\frac{n}{2}} \int_{\mathbb{R}^{n}} T\left(P L_{\varepsilon \alpha}\right)(x) \overline{\left(Q L_{\varepsilon \beta}\right)(x)} d x=\int_{\mathbb{T}^{n}} S(P)(x) \overline{Q(x)} d x .
$$

The above lemma is different from Lemma 3.6.8. from [13] page 224 only in one aspect: as we are looking to obtain results for functions with mean value 0 , we may ask $m$ to be regulated at every point of $\mathbb{Z}^{n} \backslash\{0\}$ instead of $\mathbb{Z}^{n}$. However, the proof is the same word for word. Finally, we are able to ass the following

Theorem A.3. Suppose that $m: \mathbb{R}^{n} \backslash\{0\} \rightarrow \mathbb{C}(p, p)$-Fourier multiplier on $\mathbb{R}^{n}$ for some $p \in[1, \infty)$ and that it is regulated at every point in $\mathbb{Z}^{n} \backslash\{0\}$. Then, $\{m(k)\}_{k \in \mathbb{Z}^{n} \backslash\{0\}}$ defines a $(p, p)$-Fourier multiplier and

$$
\left\|\{m(k)\}_{k \in \mathbb{Z}^{n} \backslash\{0\}}\right\|_{M_{p}\left(\mathbb{Z}^{n}\right)} \leq\|m\|_{M_{p}\left(\mathbb{R}^{n}\right)} .
$$

Theorem A.3 is a restatement of Theorem 3.6.7. from [13] page 224 in the context of $L^{p}$ functions with mean value 0 . The proof is a consequence of the fact that the $L^{p}$-norm of a function can be expressed by duality as the supremum over all trigonometric functions with $L^{p^{\prime}}$ norm less than 1 combined with A.1. The interested reader is referred to [13] pages $224-225$ for a complete proof.

We use Theorem A.3 and Remark A.1 in order to estimate the norm of the Fourier multiplier operator on the torus

$$
\left(I d-(2 \mu+\lambda)\left(\mu \Delta_{\theta}+(\mu+\lambda) \Delta\right)^{-1} \Delta\right)
$$

whose multiplier is

$$
m\left(\xi_{1}, \xi_{2}, \xi_{3}\right)=\frac{\theta \mu\left|\xi_{3}\right|^{2}}{(2 \mu+\lambda)\left(\left|\xi_{1}\right|^{2}+\left|\xi_{2}\right|^{2}\right)+((2+\theta) \mu+\lambda)\left|\xi_{3}\right|^{2}} .
$$

According to Remark A.1 and Theorem A.3, taking in consideration that $\theta>-1$ and after some long but straightforward computations we obtain that there exists a numerical constant $C>0$ such that

$$
\|m\|_{M_{p}\left(\mathbb{Z}^{n}\right)} \leq C(1+|\theta|)|\theta| \mu \frac{(2 \lambda+\mu)}{(\lambda+\mu)^{2}} .
$$

Acknowledgments. D. Bresch and C. Burtea are supported by the SingFlows project, grant ANR18-CE40-0027 and D. Bresch is also supported by the Fraise project, grant ANR-16-CE06- 0011 of the French National Research Agency (ANR). 


\section{References}

[1] Beirão Da Veiga, H. An $L^{p}$-theory for the n-dimensional, stationary, compressible NavierStokes equations, and the incompressible limit for compressible fluids. The equilibrium solutions. Comm. Math. Phys. Vol. 109. Issue 2, 229-248, 1987.

[2] Bresch, D., And C. Burtea Global Existence of Weak Solutions for the Anisotropic Compressible Stokes System. arXiv:190\%.09171, 2019.

[3] Bresch, D., And P.-E. Jabin.Global existence of weak solutions for compresssible Navier-Stokes equations: Thermodynamically unstable pressure and anisotropic viscous stress tensor. Annals of Math, Vol. 188, Issue 2, 577-684, 2018.

[4] Bresch, D. And P.-E. Jabin Global weak solutions of PDEs for compressible media: A compactness criterion to cover new physical situations. Shocks, Singularities and Oscillations in Nonlinear Optics and Fluid Mechanics, Springer INdAM Vol. 17, 33-54, 2017.

[5] BŘezina, J.,And A. Novotný. On weak solutions of steady Navier-Stokes equations for monoatomicgas. Comment. Math. Univ. Carolin, Vol. 49, No. 4, 611-632, 2008.

[6] Dou, C., Jiang, F., Jiang, S., And Y.F. Yang. Existence of strong solutions to the steady Navier-Stokes equations for a compressible heat-conductive fluid with large forces. J. Math. Pures et Appl., Vol. 103, No. 5, 1163-1197, 2015.

[7] Eringen, A.C. On nonlocal fluid mechanics. Intern. J. Engineering Science, Vol. 10, No. 6, 561-575, 1972.

[8] ERingen, A.C. Nonlocal continuum field theories. Springer, 2002.

[9] Feireisl, E. On compactness of solutions to the compressible isentropic Navier-Stokes equations when the density is not square integrable. Commentationes Mathematicae Universitatis Carolinae, Vol. 42, No. 1, 83-98, 2001.

[10] Feireisl, E., And A. Novotný. Stationary solutions to the compressible Navier-Stokes system with general boundary conditions. Ann. IHP C, Analyse nonlinéaire. Vol. 35. No. 6, 2018.

[11] Frehse, J., Steinhauer, M. and W. Weigant. The Dirichlet problem for steady viscous compressible flow in three dimensions. J. Math. Pures Appl., Vol. 97, No 2, 85-97, 2012.

[12] Gilbarg, D., And N.S. Trudinger. Elliptic partial differential equations of second order. Springer, 2015.

[13] Grafakos, L.. Classical fourier analysis. Vol. 1. New York: Springer, 2008.

[14] Hoff, D., And J. Smoller. Solutions in the large for certain nonlinear parabolic systems. Ann. IHP (C) Non Linear Analysis, Vol. 2. No. 3. Elsevier Masson, 1985.

[15] Jiang, S., And C. Zhou. Existence of weak solutions to the three-dimensional steady compressible Navier-Stokes equations. Ann. IHP Analyse non linéaire, Vol. 28, No. 4, 485-498, 2011.

[16] Lions, P.-L. Mathematical Topics in Fluid Mechanics: Volume 2: Compressible Models. Oxford University Press, 1998.

[17] Novo, S., And A. Novotný. On the existence of weak solutions to the steady compressible Navier-Stokes equations when the density is not square integrable. J. Math. Kyoto Univ., Vol. 42, No.3, 531-550, 2002.

[18] Novo, S., And A. NovotnÝ. A remark on the smoothness of bounded regions filled with a steady compressible and isentropic fluid. Applications of Mathematics, Vol. 50, No. 4, 331-339, 2005. 
[19] Novo, S., Novotný, A., And M. Pokorný. Steady compressible Navier-Stokes equations in domains with non-compact boundaries. Math. Methods Appl. Sciences, Vol. 28, No. 12, 1445-1479, 2005 .

[20] NovotnÝ, A., And I. StrašKraba. Introduction to the mathematical theory of compressible flow, Oxford University Press, 2004.

[21] Padula, M. Existence and uniqueness for viscous steady compressible motions. Arch. Rational Mech. Anal., Vol. 97, No. 2, 89-102, 1987.

[22] Pedlosky, J. Geophysical fluid dynamics. Springer 2013.

[23] Plotnikov, P.I., And J. Sokolowski. Stationary solutions of Navier-Stokes equations for diatomic gases. Russian Mathematical Surveys Vol 62, No. 3, 2007.

[24] Plotnikov, P.I., And W. Weigant. Steady 3D viscous compressible flows with adiabatic exponent $\gamma \in(1, \infty)$. J. Math. Pures Appl. Vol. 104, No. 1, pp. 58-82, 2015.

[25] Pokorný, M., And P.B. Mucha. 3D steady compressible Navier-Stokes equations. DCDS-S Vol 1, No. 1, 151-163, 2008.

[26] Serre, D. Variations de grande amplitude pour la densité d'un fluide visqueux compressible. Physica D: Nonlinear Phenomena Vol. 48, No. 1, 113-128, 1991.

[27] VAlli, A., AND W.M. ZaJACZKOWski. Navier-Stokes equations for compressible fluids: global existence and qualitative properties of the solutions in the general case. Comm. Math. Phys. Vol. 103, No. 2, 259-296, 1986. 